ESAIM: M2AN 51 (2017) 1561-1581

DOI: $10.1051 / \mathrm{m} 2 \mathrm{an} / 2016075$
ESAIM: Mathematical Modelling and Numerical Analysis

www.esaim-m2an.org

\title{
AN EDGE-BASED SCHEME ON POLYHEDRAL MESHES FOR VECTOR ADVECTION-REACTION EQUATIONS
}

\author{
Pierre Cantin ${ }^{1,2}$ And Alexandre Ern ${ }^{1}$
}

\begin{abstract}
We devise and analyze an edge-based scheme on polyhedral meshes to approximate a vector advection-reaction problem. The well-posedness of the discrete problem is analyzed first under the classical positivity hypothesis of Friedrichs' systems that requires a lower bound on the lowest eigenvalue of some tensor depending on the model parameters. We also prove stability when the lowest eigenvalue is null or even slightly negative if the mesh size is small enough. A priori error estimates are established for solutions in $\boldsymbol{W}^{1, q}(\Omega)$ with $q \in\left(\frac{3}{2}, 2\right]$. Numerical results are presented on threedimensional polyhedral meshes.
\end{abstract}

Mathematics Subject Classification. 65N12, 65N15, 76R99, 76D07, 76W05.

Received June 1, 2016. Revised November 21, 2016. Accepted November 28, 2016.

\section{INTRODUCTION}

Let $\Omega$ be a polyhedral domain of $\mathbb{R}^{d}$ with $d=3$ and consider a polyhedral mesh of $\Omega$. We use boldface fonts for $\mathbb{R}^{d}$ or $\mathbb{R}^{d \times d}$-valued quantities. The purpose of this paper is to devise an approximation, using scalar degrees of freedom (dofs) attached to the edges of a mesh, of the $\mathbb{R}^{d}$-valued function $\boldsymbol{u}$ solving the vector advection-reaction problem:

$$
\begin{aligned}
\nabla(\boldsymbol{\beta} \cdot \boldsymbol{u})+(\nabla \times \boldsymbol{u}) \times \boldsymbol{\beta}+\boldsymbol{\mu} \boldsymbol{u} & =\boldsymbol{s} \quad \text { a.e. in } \Omega, \\
\boldsymbol{u} & =\boldsymbol{u}_{D} \text { a.e. on } \partial \Omega^{-} .
\end{aligned}
$$

The $\mathbb{R}^{d}$-valued advective field $\boldsymbol{\beta}$ is assumed to be Lipschitz continuous in $\Omega$ and the $\mathbb{R}^{d \times d}$-valued reaction tensor $\boldsymbol{\mu}$ is assumed to be bounded in $\Omega$. The subset $\partial \Omega^{-} \subset \partial \Omega$ denotes the inflow part of the boundary where $\boldsymbol{\beta} \cdot \boldsymbol{n}<0$ with $\boldsymbol{n}$ the unit outward normal to $\Omega$.

The model problem (1.1) is encountered in various situations. For example, it models the static advection of a magnetic field ( $\boldsymbol{u}$ here) by a moving plasma of velocity $\boldsymbol{\beta}$ and of anisotropic conductivity $\boldsymbol{\mu}$. In the context of differential geometry, the operator $\nabla(\boldsymbol{\beta} \cdot \boldsymbol{u})+(\nabla \times \boldsymbol{u}) \times \boldsymbol{\beta}$ is the proxy of the Lie derivative of a differential 1-form (also called circulation) in $\mathbb{R}^{3}$ (see Abraham et al. [1] or Heumann [18]). The Lie derivative describes more generally the advection along the vector field $\boldsymbol{\beta}$ of a differential form on a manifold. The model problem (1.1)

\footnotetext{
Keywords and phrases. Vector advection-reaction problems, polyhedral meshes, Friedrichs' assumptions, quasi-optimal a priori error estimates.

1 Université Paris-Est, CERMICS (ENPC), 77455 Marne la Vallée cedex 2, France.

pircantin@gmail.com; alexandre.ern@enpc.fr

2 EDF R\&D, 6 quai Watier, BP 49, 78401 Chatou, France.
} 
is also relevant to study, in the advection-dominant regime, the advection-diffusion of a $\mathbb{R}^{d}$-valued field, which is one the building blocks of the Oseen problem or of the magneto-hydrodynamic problem. Using vector calculus rules, we observe that

$$
\begin{aligned}
\nabla(\boldsymbol{\beta} \cdot \boldsymbol{u}) & =(\nabla \boldsymbol{\beta})^{\mathrm{T}} \boldsymbol{u}+(\nabla \boldsymbol{u})^{\mathrm{T}} \boldsymbol{\beta}, \\
(\nabla \times \boldsymbol{u}) \times \boldsymbol{\beta} & =(\nabla \boldsymbol{u}) \boldsymbol{\beta}-(\nabla \boldsymbol{u})^{\mathrm{T}} \boldsymbol{\beta},
\end{aligned}
$$

where we have denoted $\nabla \boldsymbol{v}$ the Jacobian matrix of $\boldsymbol{v}: \Omega \rightarrow \mathbb{R}^{3}$ such that its $(i, j)$-th component is $\partial_{j} \boldsymbol{v}_{i}$. As a result, combining the two above equations yields $\nabla(\boldsymbol{\beta} \cdot \boldsymbol{u})+(\nabla \times \boldsymbol{u}) \times \boldsymbol{\beta}=(\nabla \boldsymbol{u}) \boldsymbol{\beta}+(\nabla \boldsymbol{\beta})^{\mathrm{T}} \boldsymbol{u}$, so that the particular choice $\boldsymbol{\mu}=-(\nabla \boldsymbol{\beta})^{\mathrm{T}}$ yields the pure advection problem (with the more usual writing $(\nabla \boldsymbol{u}) \boldsymbol{\beta}=(\boldsymbol{\beta} \cdot \nabla) \boldsymbol{u}$ in this context):

$$
\begin{aligned}
(\boldsymbol{\beta} \cdot \nabla) \boldsymbol{u} & =s \quad \text { a.e. in } \Omega, \\
\boldsymbol{u} & =\boldsymbol{u}_{D} \text { a.e. on } \partial \Omega^{-} .
\end{aligned}
$$

Edge-based schemes, that is, schemes using one scalar degree of freedom (dof) per mesh edge, are rarely addressed in the literature despite the fact that they are the natural way to discretize differential 1-forms, such as the electric field in electromagnetism or the flow velocity in fluid mechanics. For the Maxwell and the Stokes problem respectively, we mention for example the work of Zaglmayr [25] and that of Girault [17] using Nédélec edge elements. In the context of our problem (1.1), Heumann and Hiptmair proposed in [19] an $\mathbf{H}(\operatorname{curl} ; \Omega)$-conforming discretization of arbitrary order using Nédélec edge elements on simplicial meshes with a stabilization term in the spirit of the discontinuous Galerkin method (see Lesaint and Raviart [22], or Johnson and Pitkäranta [20]). In a different context and motivated by the discretization of the Lie derivative of a 1-form, we mention the Ph.D. thesis of Palha [24] approximating on square meshes a problem similar to (1.1) with the spectral element method. Based on the work of Bossavit [7], Mullen et al. also studied in [23] an approximation of (1.1) by extruding the edges of a simplicial mesh along the vector field $\boldsymbol{\beta}$. All of the above schemes are devised on either simplicial or tensor-product meshes.

The first salient contribution of this work is to devise an edge-based scheme to approximate the model problem (1.1) on polyhedral meshes. The advantage of considering polyhedral meshes is multifold; it allows for more flexibility when meshing a complex geometry, it provides a natural framework to handle non-matching mesh refinement and mesh coarsening by cell agglomeration, and it may even yield lower computational costs and better accuracy compared to the case of the simplicial meshes (see [3] Bonelle's Ph.D. thesis). The analysis framework for our scheme hinges on the notions of reduction and reconstruction maps as, e.g., in the mimetic approach of Kreeft et al. in [21], see also Gerritsma [16], or the Compatible Discrete Operator (CDO) approach of Bonelle and Ern $[5,6]$. In particular, we consider a reconstruction map defining piece-wise constant vectorvalued functions on an edge-based diamond partition of each mesh cell. This map has been introduced by Codecasa et al. in [10] and has been recently revisited in the context of CDO schemes in [5]. The novelty here is to perform the stability analysis in $\boldsymbol{L}^{q}$-spaces for $q \in[1, \infty)$ and to prove a quasi-local consistency result by composing the reconstruction map on the right with a novel reduction map à la Clément that is stable for all integrable functions on a macro cell collecting all diamonds attached to the cell edges. This technique is key to establish an $\mathcal{O}\left(h^{q}\right)$ convergence rate as soon as the weak solution belongs to $\boldsymbol{W}^{1, q}(\Omega)$ with $q \in\left(\frac{3}{2}, 2\right]$ without invoking a more stringent regularity assumption.

The second salient contribution of this work is to extend the well-posedness analysis at the discrete level to the non-coercive case. Specifically, we introduce an extended hypothesis on the problem coefficients (the fields $\boldsymbol{\beta}$ and $\boldsymbol{\mu}$ ) that allows one to go beyond the classical (and somewhat restrictive) assumption à la Friedrichs requiring the positivity of the minimal eigenvalue of the symmetric tensor

$$
\boldsymbol{\sigma}_{\boldsymbol{\beta}, \boldsymbol{\mu}}:=\left(\nabla \boldsymbol{\beta}+\nabla \boldsymbol{\beta}^{\mathrm{T}}\right)-(\nabla \cdot \boldsymbol{\beta}) \mathbf{I d}+\left(\boldsymbol{\mu}+\boldsymbol{\mu}^{\mathrm{T}}\right) \Omega \rightarrow \mathbb{R}^{3 \times 3} .
$$

Under this hypothesis, the well-posedness of the discrete problem classically hinges on a coercivity argument. However, this assumption is somehow restrictive; e.g. , the basic case of a constant vector field $\boldsymbol{\beta}$ with no reaction 
term does not fulfill this hypothesis. Motivated by our recent work [8] related to scalar advection-diffusion problem (see also the work of Deuring et al. in [11] for face-based finite volume schemes), we propose to extend the analysis to the non-coercive case where the minimal eigenvalue $\lambda_{b}$ can take null or slightly negative values.

Even if our analysis is presented here for our scheme, we emphasize that the main idea can be adapted to other schemes, such as Nédélec edge elements. We denote $\lambda_{b}$ the minimal eigenvalue of $\boldsymbol{\sigma}_{\boldsymbol{\beta}, \boldsymbol{\mu}}$ over the domain $\Omega$, i.e.

$$
\lambda_{b}=\underset{\boldsymbol{x} \in \Omega}{\operatorname{ess} \inf _{\boldsymbol{y} \in \mathbb{R}^{d}}} \min _{\boldsymbol{\beta}, \boldsymbol{\mu}(\boldsymbol{x}) \boldsymbol{y}, \boldsymbol{y})_{\ell^{2}}},
$$

where $|\cdot|_{\ell^{2}}$ denotes the Euclidean norm induced by the Euclidean inner-product $(\cdot, \cdot)_{\ell^{2}}$ in $\mathbb{R}^{d}$. Assuming that $\boldsymbol{s} \in \boldsymbol{L}^{2}(\Omega), \boldsymbol{u}_{D} \in \boldsymbol{L}^{2}(|\boldsymbol{\beta} \cdot \boldsymbol{n}| ; \partial \Omega)$ and that $\operatorname{dist}\left(\partial \Omega^{-}, \partial \Omega^{+}\right)>0$ (with $\partial \Omega^{+}$the outflow part of the boundary), we infer from Ern and Guermond in [14] that the problem (1.1) is well-posed in the graph space $\boldsymbol{V}_{\boldsymbol{\beta}}(\Omega)=\{\boldsymbol{v} \in$ $\left.\boldsymbol{L}^{2}(\Omega) \mid(\boldsymbol{\beta} \cdot \nabla) \boldsymbol{v} \in \boldsymbol{L}^{2}(\Omega)\right\}$ if the fields $\boldsymbol{\beta}$ and $\boldsymbol{\mu}$ satisfy the following hypothesis:

(H1) $\lambda_{b}>0$. We define the reference time $\tau=\lambda_{b}^{-1}$.

(H2) $-C_{\lambda}<\lambda_{b} \leq 0$, where $C_{\lambda}>0$ is a constant independent the mesh size, and there exists a potential $\zeta \in W^{1, \infty}(\Omega)$ satisfying $\zeta \geq 1$ and $\operatorname{ess} \inf _{\Omega}(-\boldsymbol{\beta} \cdot \nabla \zeta)>0$. We define the reference time $\tau=\left(\operatorname{ess} \inf _{\Omega}(-\boldsymbol{\beta} \cdot \nabla \zeta)\right)^{-1}$.

In the case of a continuously differentiable vector field $\boldsymbol{\beta} \in \boldsymbol{C}^{1}(\Omega)$, the existence of the potential $\zeta$ is proved by Devinatz et al. in ([12], Lem. 2.3) by considering the Cauchy problem $d_{t} \boldsymbol{x}(t)=\boldsymbol{\beta}(\boldsymbol{x}(t)), \boldsymbol{x}(0)=\boldsymbol{x}_{0} \in \Omega$ when the solution remains in the domain $\Omega$ for a finite time only. As a result, the hypothesis $(\mathbf{H 2})$ is satisfied if the vector field $\boldsymbol{\beta}$ has no closed curves and no stationary points in $\Omega$. The analysis of our polyhedral edge-based scheme under this second hypothesis (H2) differs since the key idea is now to bound, at first-order in the mesh size, the commutator between the reconstruction map and the multiplication by the potential $\zeta$. Using this technique, we can prove inf-sup stability (and infer the same convergence rates as above) as soon as the mesh size is smaller than a reference length that linearly depends on the quantity $\left\|\nabla \boldsymbol{\beta}^{\mathrm{T}}+\boldsymbol{\mu}\right\|_{\boldsymbol{L}^{\infty}(\Omega)}^{-1}$. In particular, for the advective problem (1.3) (where $\boldsymbol{\mu} \equiv-\nabla \boldsymbol{\beta}^{\mathrm{T}}$ ), inf-sup stability holds with no restriction on the mesh size.

This paper is organized as follows. In Section 2, we introduce the notation and the analysis tools on polyhedral meshes. In Section 3, we introduce the edge-based reconstruction map and we present the numerical scheme with dofs attached to edges. In Section 4, we state the main analytic results, namely, stability under hypothesis (H1) or (H2), boundedness and a priori error estimates delivering quasi-optimal decay rates for solutions in $\boldsymbol{W}^{1, q}(\Omega)$ with $q \in\left(\frac{3}{2}, 2\right]$. The proofs are postponed to Section 6 to facilitate the reading. Finally, we present in Section 5 numerical results on three-dimensionnal polyhedral meshes. A natural perspective for this work is to use the present scheme to discretize the advective operator in the Oseen (and Navier-Stokes) equations, while using the CDO scheme of [6] to discretize the Stokes operator in curl-curl formulation.

\section{NotATION AND ANALYSIS TOOLS ON POLYHEDRAL MESHES}

We consider a general mesh $\mathrm{M}$ of $\Omega \subset \mathbb{R}^{d}$ with $d=3$, composed of polyhedral cells $c \in \mathrm{C}$ (3-cells), planar faces $f \in \mathrm{F}$ (2-cells), straight edges $e \in \mathrm{E}$ (1-cells), and vertices $v \in \mathrm{V}$ (0-cells). We collect the interior faces in the set $\mathrm{F}^{\circ}=\left\{f=\partial c \cap \partial c^{\prime} \mid c \neq c^{\prime}\right.$ and $\left.c, c^{\prime} \in \mathrm{C}\right\}$, and we define $\mathrm{F}^{\partial}=\mathrm{F} \backslash \mathrm{F}^{\circ}$ the set collecting boundary faces. For any $\mathrm{A}, \mathrm{X} \in\{\mathrm{V}, \mathrm{E}, \mathrm{F}, \mathrm{C}\}$, we define the subset $\mathrm{X}_{a}$ with $a \in \mathrm{A}$ as $\{x \in \mathrm{X} \mid a \subset \partial x\}$ if the dimension of $a$ is smaller than that of the elements of $\mathrm{X}$ and as $\mathrm{X}_{a}=\{x \in \mathrm{X} \mid x \subset \partial a\}$ otherwise. For example, the set $\mathrm{C}_{e}=\{c \in \mathrm{C} \mid e \subset \partial c\}$ collects all the mesh cells containing the edge $e$, whereas the set $\mathrm{E}_{c}=\{e \in \mathrm{E} \mid e \subset \partial c\}$ collects all the mesh edges contained in the cell $c$, and so on. For any geometric entity $x$, we denote $|x|$ its Hausdorff measure of appropriate dimension. In this paper, we assume mesh regularity in the sense that

- The mesh $\mathrm{M}:=\{\mathrm{V}, \mathrm{E}, \mathrm{F}, \mathrm{C}\}$ defines a cellular complex (see Christiansen [9]), i.e. the boundary of any $k$-cell, $1 \leq k \leq d$ (recall $d=3$ ), is composed of a uniformly finite number of $(k-1)$-cells in M.

- Faces and cells are star-shaped with respect to their barycenters. 

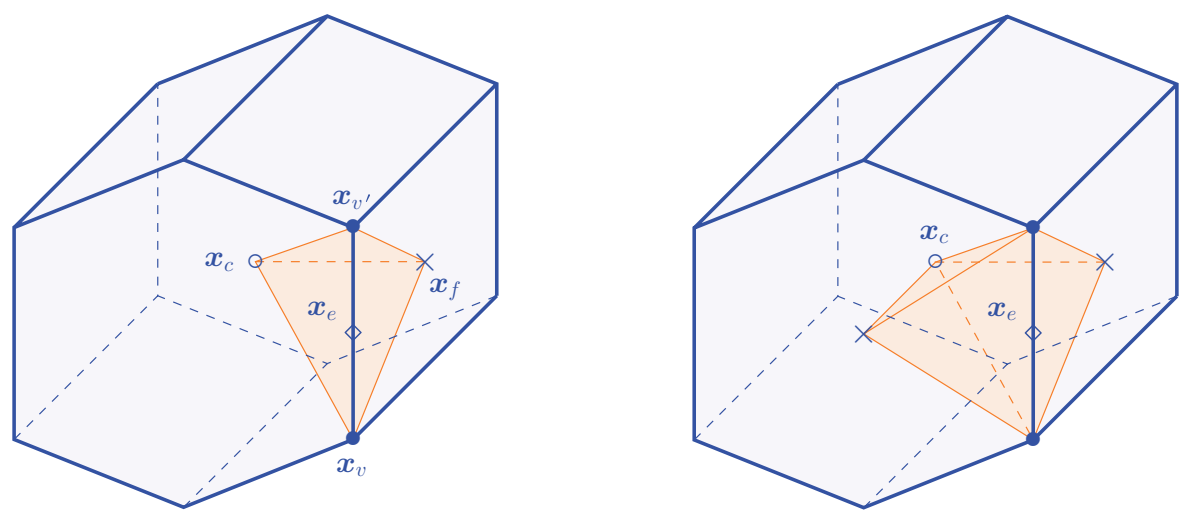

Figure 1. Left panel: Tetrahedron $\left[\boldsymbol{x}_{v}, \boldsymbol{x}_{v^{\prime}}, \boldsymbol{x}_{f}, \boldsymbol{x}_{c}\right]$. Right panel: Local diamond $\mathfrak{p}_{e, c}$.

- Let $\boldsymbol{x}_{v}$ denote the coordinates of $v \in \mathrm{V}$ in $\mathbb{R}^{d}$. Let $\boldsymbol{x}_{f}$ and $\boldsymbol{x}_{c}$ denote the coordinates of the barycenters of $f \in$ $\mathrm{F}$ and $c \in \mathrm{C}$, respectively, in $\mathbb{R}^{d}$. Then, the simplicial sub-mesh composed of the tetrahedra $\left[\boldsymbol{x}_{v}, \boldsymbol{x}_{v^{\prime}}, \boldsymbol{x}_{f}, \boldsymbol{x}_{c}\right]$ (where $\left[\boldsymbol{x}_{1}, \ldots, \boldsymbol{x}_{k+1}\right]$ is the convex hull of the set $\left\{\boldsymbol{x}_{1}, \ldots, \boldsymbol{x}_{k+1}\right\}$ ) for all $c \in \mathrm{C}$, all $f \in \mathrm{F}_{c}$ and all $e \in \mathrm{E}_{f}$ with $e=\left[\boldsymbol{x}_{v}, \boldsymbol{x}_{v^{\prime}}\right]$ (see Fig. 1, left panel) is shape-regular in the usual sense of Ciarlet.

For every cell $c \in \mathrm{C}$, we introduce the edge-based diamond partition $\mathfrak{P}_{c}$ which plays a central role in our analysis. We define $\mathfrak{P}_{c}=\cup\left\{\mathfrak{p}_{e, c} ; e \in \mathrm{E}_{c}\right\}$ where the diamond $\mathfrak{p}_{e, c}$ is defined by

$$
\mathfrak{p}_{e, c}=\bigcup_{f \in \mathrm{F}_{c} \cap \mathrm{F}_{e}}\left[\boldsymbol{x}_{v}, \boldsymbol{x}_{v^{\prime}}, \boldsymbol{x}_{f}, \boldsymbol{x}_{c}\right] \text { with } e=\left[\boldsymbol{x}_{v}, \boldsymbol{x}_{v^{\prime}}\right],
$$

see Figure 1, right panel. Note that $\mathfrak{P}_{c}$ is composed of $\# \mathrm{E}_{c}$ diamonds and that each diamond $\mathfrak{p}_{e, c}$ is composed of two tetrahedra, since $\#\left(\mathrm{~F}_{e} \cap \mathrm{F}_{c}\right)=2$, with \# the cardinal operator. Owing to the star-shaped property of faces and cells, we have $c=\cup\left\{\mathfrak{p} ; \mathfrak{p} \in \mathfrak{P}_{c}\right\}$. The skeleton of the global partition $\mathfrak{P}=\cup\left\{\mathfrak{P}_{c} \mid c \in \mathrm{C}\right\}$ consists of the collection of all the triangular sub-faces defining the boundary of each diamond $\mathfrak{p}_{e, c}$. There are two types of sub-faces: intra-cell sub-faces attached to a cell $c \in \mathrm{C}$ and collected in the set $\mathfrak{F}_{c}=\left\{\mathfrak{f}=\partial \mathfrak{p}_{e, c} \cap \partial \mathfrak{p}_{e^{\prime}, c} \mid e \neq\right.$ $e^{\prime}$ and $\left.e, e^{\prime} \in \mathrm{E}_{c}\right\}$ so that $\mathfrak{f} \not \subset \partial c$, (see Fig. 2, left panel) and inter-cell sub-faces attached to an interior face $f \in \mathrm{F}^{\circ}$ and collected in the set $\mathfrak{F}_{f}=\left\{\mathfrak{f}=\partial \mathfrak{p}_{e, c} \cap \partial \mathfrak{p}_{e, c^{\prime}} \mid c \neq c^{\prime}\right.$ and $\left.c, c^{\prime} \in \mathrm{C}_{f}, e \in \mathrm{E}_{f}\right\}$ (see Fig. 2, right panel). All the sub-faces are oriented by a fixed unit normal vector $\boldsymbol{n}_{\mathfrak{f}}$. For all $\mathfrak{f}=\partial \mathfrak{p}_{e, c} \cap \partial \mathfrak{p}_{e^{\prime}, c} \in \mathfrak{F}_{c}$ with $e, e^{\prime} \in \mathrm{E}_{c}$ and $\boldsymbol{n}_{\mathfrak{f}}$ pointing from $\mathfrak{p}_{e, c}$ to $\mathfrak{p}_{e^{\prime}, c}$, we define the jump and the average, respectively, as

$$
\llbracket \boldsymbol{v} \rrbracket=\boldsymbol{v}_{\mid \mathfrak{p}_{e, c}}-\boldsymbol{v}_{\mid \mathfrak{p}_{e^{\prime}, c}} \text { and }\left\{\{\boldsymbol{v}\}:=\frac{1}{2}\left(\boldsymbol{v}_{\mid \mathfrak{p}_{e, c}}+\boldsymbol{v}_{\mid \mathfrak{p}_{e^{\prime}, c}}\right) .\right.
$$

Similarly, for all $\mathfrak{f}=\partial \mathfrak{p}_{e, c} \cap \partial \mathfrak{p}_{e, c^{\prime}} \in \mathfrak{F}_{f}$ with $c, c^{\prime} \in \mathrm{C}_{f}, e \in \mathrm{E}_{f}$, and $\boldsymbol{n}_{\mathfrak{f}}$ pointing from $\mathfrak{p}_{e, c}$ to $\mathfrak{p}_{e, c^{\prime}}$, we define

$$
\llbracket \boldsymbol{v} \rrbracket:=\boldsymbol{v}_{\mid \mathfrak{p}_{e, c}}-\boldsymbol{v}_{\mid \mathfrak{p}_{e, c^{\prime}}} \text { and }\left\{\{\boldsymbol{v}\}:=\frac{1}{2}\left(\boldsymbol{v}_{\mid \mathfrak{p}_{e, c}}+\boldsymbol{v}_{\mid \mathfrak{p}_{e, c^{\prime}}}\right)\right. \text {. }
$$

We denote $|\cdot|_{\ell^{2}}$ the Euclidean and the Frobenius norm on $\mathbb{R}^{d}$ and $\mathbb{R}^{d \times d}$, respectively. For every set $\omega \subset \bar{\Omega}$, we denote $\boldsymbol{L}^{q}(\omega)$ with $q \in[1, \infty]$ the Banach space of $\mathbb{R}^{d}$ or $\mathbb{R}^{d \times d}$-valued functions $\boldsymbol{v}$ such that $\|\boldsymbol{v}\|_{\boldsymbol{L}^{q}(\omega)}:=$ $\left\||\boldsymbol{v}|_{\ell^{2}}\right\|_{L^{q}(\omega)}<\infty$.

Lemma 2.1 (Mutliplicative trace inequality). There exists $C_{\mathrm{T}}>0$ such that

$$
\|\boldsymbol{v}\|_{\boldsymbol{L}^{q}(\mathfrak{f})} \leq C_{\mathrm{T}}\|\boldsymbol{v}\|_{\boldsymbol{L}^{q}(\mathfrak{p})}^{1-\frac{1}{q}}\left(h_{c}^{-\frac{1}{q}}\|\boldsymbol{v}\|_{\boldsymbol{L}^{q}(\mathfrak{p})}^{\frac{1}{q}}+|\boldsymbol{v}|_{\boldsymbol{W}^{1, q}(\mathfrak{p})}^{\frac{1}{q}}\right)
$$

for all $c \in \mathrm{C}$ with $h_{c}$ the diameter of $c$, all $\mathfrak{p} \in \mathfrak{P}_{c}$, all $\mathfrak{f} \in \partial \mathfrak{p}$ and all $\boldsymbol{v} \in \boldsymbol{W}^{1, q}(\mathfrak{p})$ with $q \in[1, \infty]$. 

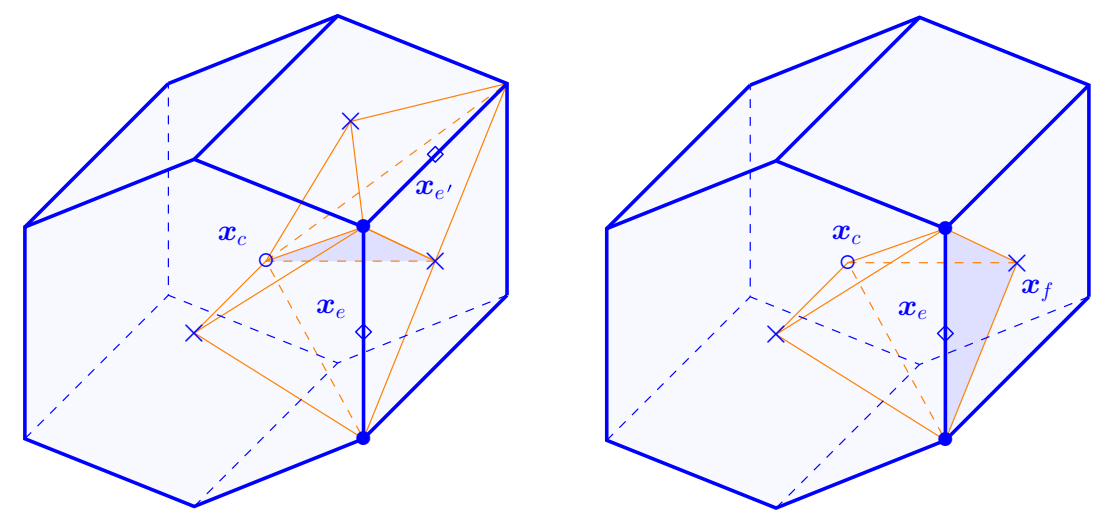

FiguRE 2. In blue. Left: Intra-cell sub-face $\mathfrak{f}=\partial \mathfrak{p}_{e, c} \cap \partial \mathfrak{p}_{e^{\prime}, c} \in \mathfrak{F}_{c}$. Right: Inter-cell sub-face $\mathfrak{f}=\partial \mathfrak{p}_{e, c} \cap \partial \mathfrak{p}_{e, c^{\prime}} \in \mathfrak{F}_{f}$. (Color online)

Proof. Observing that $\mathfrak{p} \subset \mathfrak{P}_{c}$ is composed of two tetrahedra connected by a sub-face $\mathfrak{f} \in \mathfrak{F}_{c}$, this result follows proceeding as in Ern and Guermond [15].

\section{DiscRete SCHEME}

\subsection{Degrees of freedom}

We consider an approximation of the continuous problem (1.1) with scalar dofs attached to edges. We denote $\mathcal{E} \equiv \mathbb{R}^{\# \mathrm{E}}$ the linear space collecting these dofs and we denote $\mathrm{v}_{e}$ the entry of $\mathrm{v} \in \mathcal{E}$ attached to the edge $e \in \mathrm{E}$. We additionally introduce the linear space $\mathcal{E}_{c}$ collecting the dofs attached to the subset $\mathrm{E}_{c}$ for all $c \in \mathrm{C}$. We denote $\vee$ a generic element of $\mathcal{E}$ or $\mathcal{E}_{c}$.

\subsection{Reconstruction map}

The global reconstruction map $\mathbf{L}_{\mathcal{E}}$ is defined locally, so that $\mathbf{L}_{\mathcal{E}}(\mathrm{v})_{\mid c}=\mathbf{L}_{\mathcal{E}_{c}}(\mathrm{v})$, for all $c \in \mathrm{C}$. The local reconstruction map $\mathbf{L}_{\mathcal{E}_{c}}: \mathcal{E}_{c} \rightarrow \mathbb{P}_{0}\left(\mathfrak{P}_{c} ; \mathbb{R}^{d}\right)$, where $\mathbb{P}_{0}\left(\mathfrak{P}_{c} ; \mathbb{R}^{d}\right)$ is composed of piece-wise constant $\mathbb{R}^{d}$-valued polynomials over the diamond partition $\mathfrak{P}_{c}$, is such that

$$
\mathbf{L}_{\mathcal{E}_{c}}(\mathrm{v})(\boldsymbol{x}):=\sum_{e \in \mathrm{E}_{c}} \mathrm{v}_{e} \boldsymbol{\ell}_{e, c}(\boldsymbol{x}), \quad \forall \mathrm{v} \in \mathcal{E}_{c}, \quad \forall \boldsymbol{x} \in c,
$$

where for all $e \in \mathrm{E}_{c}$, the basis function $\boldsymbol{\ell}_{e, c} \in \mathbb{P}_{0}\left(\mathfrak{P}_{c} ; \mathbb{R}^{d}\right)$, is defined by

$$
\left.\boldsymbol{\ell}_{e, c}\right|_{\mathfrak{p}_{e^{\prime}, c}}=\left(\mathbf{I d}-\frac{\tilde{\boldsymbol{f}}_{c}\left(e^{\prime}\right) \otimes \boldsymbol{e}^{\prime}}{d\left|\mathfrak{p}_{e^{\prime}, c}\right|}\right) \frac{\tilde{\boldsymbol{f}}_{c}(e)}{|c|}+\frac{\tilde{\boldsymbol{f}}_{c}(e)}{d\left|\mathfrak{p}_{e, c}\right|} \delta_{e, e^{\prime}}, \quad \forall e^{\prime} \in \mathrm{E}_{c}
$$

and $\delta_{e, e^{\prime}}$ is the Kronecker symbol equal to 1 if $e=e^{\prime}$ and 0 otherwise. Moreover, for all $e \in \mathrm{E}, \boldsymbol{t}_{e}$ is a fixed unit tangent vector to $e$, such that $\boldsymbol{e}=|e| \boldsymbol{t}_{e}$, and $\tilde{\boldsymbol{f}}_{c}(e)=\int_{\tilde{f}_{c}(e)} \boldsymbol{n}_{\tilde{f}_{c}(e)}$ where the dual face $\tilde{f}(e)$ is composed of two elementary triangles

$$
\tilde{f}_{c}(e)=\bigcup_{f \in \mathrm{F}_{c} \cap \mathrm{F}_{e}}\left[\boldsymbol{x}_{e}, \boldsymbol{x}_{f}, \boldsymbol{x}_{c}\right]
$$

see Figure 3, and where $\boldsymbol{n}_{\tilde{f}_{c}(e)}$ is the unit normal vector to $\tilde{f}_{c}(e)$ satisfying $\boldsymbol{n}_{\tilde{f}_{c}(e)} \cdot \boldsymbol{t}_{e} \geq 0$. The basis functions $\boldsymbol{\ell}_{e, c}$ were first considered in the context of the Discrete Geometric Approach by Codecasa et al. [10] and were recently 


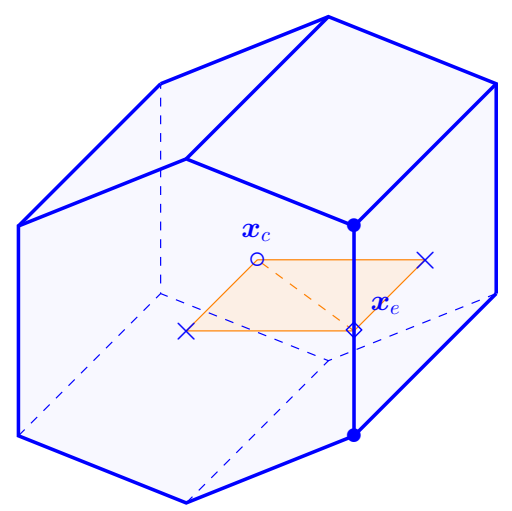

Figure 3. Local dual face $\tilde{f}_{c}(e)$.

revisited by Bonelle and Ern in $[5,6]$ to build Hodge operators within the CDO framework. They satisfy the following properties:

$(\boldsymbol{\ell} 1)$ [Unisolvence] For all $e, e^{\prime} \in \mathrm{E}_{c}, \boldsymbol{\ell}_{e, c}(\boldsymbol{x}) \cdot \boldsymbol{e}^{\prime}=\delta_{e, e^{\prime}}$ for all $\boldsymbol{x} \in \mathfrak{p}_{e^{\prime}, c}$.

$(\ell 2)\left[\right.$ Primal $\mathbb{P}_{0}$-consistency] $\sum_{e \in \mathrm{E}_{c}} \boldsymbol{\ell}_{e, c}(\boldsymbol{x}) \otimes \boldsymbol{e}=\mathbf{I d}$ for all $\boldsymbol{x} \in c$.

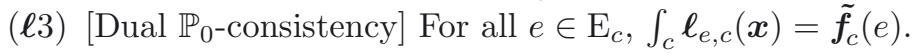

The property $(\ell 1)$ relies on the geometric relation $\left|\mathfrak{p}_{e, c}\right|=\frac{1}{d} \tilde{f}_{c}(e) \cdot \boldsymbol{e}$ whereas the property $(\ell 2)$ results from the geometric relation $\sum_{e \in \mathrm{E}_{c}} \boldsymbol{e} \otimes \tilde{\boldsymbol{f}}_{c}(e)=\sum_{e \in \mathrm{E}_{c}} \tilde{\boldsymbol{f}}_{c}(e) \otimes \boldsymbol{e}=|c| \mathbf{I d}$.

\subsection{Discrete scheme}

The discrete scheme is formulated using the global bilinear form $\mathbb{A}_{\boldsymbol{\beta}, \boldsymbol{\mu}}: \mathcal{E} \times \mathcal{E} \rightarrow \mathbb{R}$ such that

$$
\mathbb{A}_{\boldsymbol{\beta}, \boldsymbol{\mu}}(\mathrm{u}, \mathrm{v})=\mathrm{A}_{\boldsymbol{\beta}, \boldsymbol{\mu}}(\mathrm{u}, \mathrm{v})+\mathrm{A}_{(\boldsymbol{\beta} \cdot \boldsymbol{n})^{-}}^{\partial}(\mathrm{u}, \mathrm{v}),
$$

where $A_{\boldsymbol{\beta}, \boldsymbol{\mu}}$ approximates (1.1a) and $\mathrm{A}_{(\boldsymbol{\beta} \cdot \boldsymbol{n})^{-}}$- weakly enforces the boundary condition (1.1b). The bilinear form $\mathrm{A}_{\boldsymbol{\beta}, \boldsymbol{\mu}}: \mathcal{E} \times \mathcal{E} \rightarrow \mathbb{R}$ is composed of three bilinear forms also defined on $\mathcal{E} \times \mathcal{E}$ :

$$
A_{\beta, \mu}(u, v):=g_{\beta, \mu}(u, v)+n_{\beta}(u, v)+s_{\beta}(u, v) .
$$

The bilinear form $\mathbf{g}_{\boldsymbol{\beta}, \boldsymbol{\mu}}$ is assembled cell-wise as

$$
\mathrm{g}_{\boldsymbol{\beta}, \boldsymbol{\mu}}(\mathrm{u}, \mathrm{v})=\sum_{c \in \mathrm{C}} \mathrm{g}_{\boldsymbol{\beta}, \boldsymbol{\mu} ; c}(\mathrm{u}, \mathrm{v}),
$$

and each local bilinear form $\boldsymbol{g}_{\boldsymbol{\beta}, \boldsymbol{\mu} ; c}$ results from the standard Galerkin approximation of (1.1a) in $c$ using the reconstruction map $\mathrm{L}_{\mathcal{E}_{c}}$ :

$$
\mathrm{g}_{\boldsymbol{\beta}, \boldsymbol{\mu} ; c}(\mathrm{u}, \mathrm{v})=\sum_{\mathfrak{p} \in \mathfrak{P}_{c}} \int_{\mathfrak{p}}\left(\nabla\left(\boldsymbol{\beta} \cdot \mathbf{L}_{\mathcal{E}_{c}}(\mathrm{u})\right)+\left(\nabla \times \mathbf{L}_{\mathcal{E}_{c}}(\mathrm{u})\right) \times \boldsymbol{\beta}\right) \cdot \mathbf{L}_{\mathcal{E}_{c}}(\mathrm{v})+\int_{c} \boldsymbol{\mu} \mathbf{L}_{\mathcal{E}_{c}}(\mathrm{u}) \cdot \mathbf{L}_{\mathcal{E}_{c}}(\mathrm{v}) .
$$

Using the identities (1.2) and since $\mathbf{L}_{\mathcal{E}_{c}}(\mathrm{v})$ is piece-wise constant, we can reformulate this expression as

$$
\mathrm{g}_{\boldsymbol{\beta}, \boldsymbol{\mu} ; c}(\mathrm{u}, \mathrm{v})=\int_{c}\left(\nabla \boldsymbol{\beta}^{\mathrm{T}}+\boldsymbol{\mu}\right) \mathbf{L}_{\mathcal{E}_{c}}(\mathrm{u}) \cdot \mathbf{L}_{\mathcal{E}_{c}}(\mathrm{v}) .
$$


Because $\mathbf{L}_{\mathcal{E}_{c}}(\mathrm{v})$ jumps across inter-cell and intra-cell sub-faces, we also consider the bilinear form $\mathrm{n}_{\boldsymbol{\beta}}$ such that

$$
\mathrm{n}_{\boldsymbol{\beta}}(\mathrm{u}, \mathrm{v})=\sum_{c \in \mathrm{C}} \mathrm{n}_{\boldsymbol{\beta} ; c}(\mathrm{u}, \mathrm{v})+\sum_{f \in \mathrm{F}^{\circ}} \mathrm{n}_{\boldsymbol{\beta} ; f}(\mathrm{u}, \mathrm{v}),
$$

where the local bilinear forms $\mathrm{n}_{\boldsymbol{\beta} ; x}$ with $x=f$ or $x=c$ are defined as

$$
\mathrm{n}_{\boldsymbol{\beta} ; x}(\mathrm{u}, \mathrm{v})=-\sum_{\mathfrak{f} \in \mathfrak{F}_{x}} \int_{\mathfrak{f}}\left(\boldsymbol{\beta} \cdot \boldsymbol{n}_{\mathfrak{f}}\right) \llbracket \mathbf{L}_{\mathcal{E}}(\mathrm{u}) \rrbracket \cdot\left\{\mathbf{L}_{\mathcal{E}}(\mathrm{v})\right\},
$$

and the stabilization bilinear form $\mathrm{s}_{\boldsymbol{\beta}}$ such that

$$
\mathrm{s}_{\boldsymbol{\beta}}(\mathrm{u}, \mathrm{v})=\sum_{c \in \mathrm{C}} \mathrm{s}_{\boldsymbol{\beta} ; c}(\mathrm{u}, \mathrm{v})+\sum_{f \in \mathrm{F}^{\circ}} \mathrm{s}_{\boldsymbol{\beta} ; f}(\mathrm{u}, \mathrm{v}),
$$

where the local bilinear forms $\mathbf{s}_{\boldsymbol{\beta} ; x}$ with $x=f$ or $x=c$ are defined as

$$
\mathrm{s}_{\boldsymbol{\beta} ; x}(\mathrm{u}, \mathrm{v})=\sum_{\mathfrak{f} \in \mathfrak{F}_{x}} \int_{\mathfrak{f}}\left|\boldsymbol{\beta} \cdot \boldsymbol{n}_{\mathfrak{f}}\right| \llbracket \mathbf{L}_{\mathcal{E}}(\mathrm{u}) \rrbracket \cdot \llbracket \mathbf{L}_{\mathcal{E}}(\mathrm{v}) \rrbracket .
$$

The bilinear forms $\mathrm{n}_{\boldsymbol{\beta}}$ and $\mathrm{s}_{\boldsymbol{\beta}}$ are devised similarly to the discontinuous Galerkin method; $\mathrm{n}_{\boldsymbol{\beta}}$ corresponds to centered fluxes and $\mathrm{n}_{\boldsymbol{\beta}}+\mathrm{s}_{\boldsymbol{\beta}}$ to upwind fluxes. Finally, the Dirichlet boundary condition is weakly enforced by means of the bilinear form $\mathrm{A}_{\alpha}^{\partial}: \mathcal{E} \times \mathcal{E} \rightarrow \mathbb{R}\left(\right.$ with $\left.\alpha=(\boldsymbol{\beta} \cdot \boldsymbol{n})^{-}\right)$such that

$$
\mathrm{A}_{\alpha}^{\partial}(\mathrm{u}, \mathrm{v})=\sum_{f \in \mathrm{F}^{\partial}} \mathrm{A}_{\alpha ; f}^{\partial}(\mathrm{u}, \mathrm{v}) \text {. }
$$

The local bilinear form $\mathrm{A}_{\alpha ; f}^{\partial}$ is defined as

$$
\mathrm{A}_{\alpha ; f}^{\partial}(\mathrm{u}, \mathrm{v})=\int_{f} \alpha \mathbf{L}_{\mathcal{E}_{c_{f}}}(\mathrm{u}) \cdot \mathbf{L}_{\mathcal{E}_{c_{f}}}(\mathrm{v}),
$$

with $c_{f}$ is the unique cell containing the boundary face $f$.

The discrete scheme consists in finding $\mathrm{u} \in \mathcal{E}$ such that

$$
\mathbb{A}_{\boldsymbol{\beta}, \boldsymbol{\mu}}(\mathrm{u}, \mathrm{v})=\Sigma\left(\boldsymbol{s}, \boldsymbol{u}_{D} ; \mathrm{v}\right), \quad \forall \mathrm{v} \in \mathcal{E},
$$

with the right-hand side form $\Sigma\left(\boldsymbol{s}, \boldsymbol{u}_{D} ; \cdot\right): \mathcal{E} \rightarrow \mathbb{R}$ such that

$$
\Sigma\left(\boldsymbol{s}, \boldsymbol{u}_{D} ; \mathrm{v}\right):=\sum_{c \in \mathrm{C}} \int_{c} \boldsymbol{s} \cdot \mathbf{L}_{\mathcal{E}_{c}}(\mathrm{v})+\sum_{f \in \mathrm{F}^{\partial}} \int_{f}(\boldsymbol{\beta} \cdot \boldsymbol{n})^{-} \boldsymbol{u}_{D} \cdot \mathbf{L}_{\mathcal{E}_{c_{f}}}(\mathrm{v}) .
$$

\section{Stability AND ERROR ANALYSiS}

\subsection{Properties of the reconstruction map}

Proposition 4.1 (Stability). There exists $C_{\sharp}>0$ such that

$$
\|\mathrm{v}\|_{q, c} \leq\left\|\mathbf{L}_{\mathcal{E}_{c}}(\mathrm{v})\right\|_{L^{q}(c)} \leq C_{\sharp}\|\mathrm{v}\|_{q, c},
$$

for all $c \in \mathrm{C}$, all $\mathrm{v} \in \mathcal{E}_{c}$, all $q \in[1, \infty)$ and where

$$
\|\mathrm{v}\|_{q, c}=\left(\sum_{e \in \mathrm{E}_{c}} \frac{\left|\mathfrak{p}_{e, c}\right|}{|e|^{q}}\left|\mathrm{v}_{e}\right|^{q}\right)^{\frac{1}{q}}
$$


Remark 4.2 (Alternative definition). In lieu of (4.1), we could also consider the simpler discrete $\boldsymbol{L}^{q}$-norm given by $\|\mathrm{v}\|_{q, c}^{q}=h_{c}^{d-q} \sum_{e \in \mathrm{E}_{c}}\left|\mathrm{v}_{e}\right|^{q}$. Owing to mesh regularity, this definition is equivalent to (4.1) up to a uniform constant with respect to the mesh-size. We prefer to use (4.1) since it simplifies the proof of Proposition 4.1.

We introduce the reduction map $\mathrm{R}_{\mathcal{E}}: \boldsymbol{L}^{1}(\Omega) \rightarrow \mathcal{E}$ such that

$$
\left.\mathrm{R}_{\mathcal{E}}(\boldsymbol{v})\right|_{e}:=\frac{1}{\left|\mathfrak{p}_{e}\right|}\left(\int_{\mathfrak{p}_{e}} \boldsymbol{v} \cdot \boldsymbol{e}\right), \quad \forall e \in \mathrm{E},
$$

where $\mathfrak{p}_{e}=\cup\left\{\mathfrak{p}_{e, c} ; c \in \mathrm{C}_{e}\right\}$ is the diamond volume surrounding the edge $e$ and $\hat{c}$ is the local diamond patch $\hat{c}=$ $\cup\left\{\mathfrak{p}_{e} ; e \in \mathrm{E}_{c}\right\}$ surrounding the cell $c$; notice that $c \subsetneq \hat{c}$. We also define the local reduction map $\mathrm{R}_{\mathcal{E}_{c}}: \boldsymbol{L}^{1}(\hat{c}) \rightarrow \mathcal{E}_{c}$ from definition (4.2) for all $e \in \mathrm{E}_{c}$.

Remark 4.3 (De Rham's map). Requiring more regularity, the usual de Rham's reduction map defined by $\left.\mathrm{R}_{\mathcal{E}}(\boldsymbol{v})\right|_{e}=|e|^{-1} \int_{e} \boldsymbol{v} \cdot \boldsymbol{e}$ for every $e \in \mathrm{E}$ can be used as well, provided that $\boldsymbol{v} \in \boldsymbol{H}^{1+\epsilon}(\Omega)[15]$ or $\boldsymbol{v} \in\{\boldsymbol{w} \in$ $\left.\boldsymbol{H}^{\frac{1}{2}+\epsilon}(\Omega), \nabla \times \boldsymbol{w} \in \boldsymbol{L}^{2+\epsilon}(\Omega)\right\}[2]$ with $\epsilon>0$.

For each cell $c \in \mathrm{C}$, we denote $\mathcal{I}_{\mathcal{E}_{c}}$ the local interpolation operator obtained by composing the local reconstruction map with the local reduction map, i.e. $\mathcal{I}_{\mathcal{E}_{c}}=\mathbf{L}_{\mathcal{E}_{c}} \circ \mathbf{R}_{\mathcal{E}_{c}}$, so that $\mathcal{I}_{\mathcal{E}_{c}}: \boldsymbol{L}^{1}(\hat{c}) \rightarrow \mathbb{P}_{0}\left(\mathfrak{P}_{c} ; \mathbb{R}^{d}\right)$.

Proposition 4.4 (Consistency). For all $c \in \mathrm{C}$ and all $\boldsymbol{U} \in \mathbb{P}_{0}\left(\hat{c} ; \mathbb{R}^{d}\right)$ (so that $\boldsymbol{U}$ is a constant function in $\hat{c}$ ), we have $\mathcal{I}_{\mathcal{E}_{c}}(\boldsymbol{U})=\boldsymbol{U}_{\mid c}$.

Lemma 4.5 (Interpolation error). There exists $C_{\mathrm{INT}}>0$ such that for all $c \in \mathrm{C}$ and all $\boldsymbol{v} \in \boldsymbol{W}^{1, q}(\hat{c})$ with $q \in[1, \infty)$,

$$
\left\|\boldsymbol{v}-\mathcal{I}_{\mathcal{E}_{c}}(\boldsymbol{v})\right\|_{\boldsymbol{L}^{q}(c)} \leq C_{\mathrm{INT}} h_{c}|\boldsymbol{v}|_{\boldsymbol{W}^{1, q}(\hat{c})}
$$

and for all $\mathfrak{p} \in \mathfrak{P}_{c}$,

$$
\left\|\boldsymbol{v}-\mathcal{I}_{\mathcal{E}_{c}}(\boldsymbol{v})\right\|_{\boldsymbol{L}^{q}(\partial \mathfrak{p})} \leq C_{\mathrm{INT}} h_{c}^{1-\frac{1}{q}}|\boldsymbol{v}|_{\boldsymbol{W}^{1, q}(\hat{c})} .
$$

\subsection{Well-posedness under (H1)}

We consider the following stability norm on the edge dof space $\mathcal{E}$ :

$$
\|\mathrm{v}\|:=\left(\tau^{-1}\|\mathrm{v}\|_{2}^{2}+|\mathrm{v}|_{\partial}^{2}+|\mathrm{v}|_{\mathrm{s}}^{2}\right)^{\frac{1}{2}}
$$

where the reference time $\tau>0$ is defined by assumption $(\mathbf{H 1})$ or $(\mathbf{H} 2),\|\cdot\|_{2}^{2}=\sum_{c \in \mathrm{C}}\|\cdot\|_{2, c}^{2}$ is the discrete $\boldsymbol{L}^{2}$-norm with $\|\cdot\|_{2, c}$ defined by $(4.1),|\cdot|_{\partial}^{2}=\mathrm{A}_{|\boldsymbol{\beta} \cdot \boldsymbol{n}|}^{\partial}(\cdot, \cdot)$ is the semi-norm induced by the bilinear form $\mathrm{A}_{|\boldsymbol{\beta} \cdot \boldsymbol{n}|}$ defined by $(3.13)$, and $|\cdot|_{\mathrm{s}}^{2}:=\mathrm{s}_{\boldsymbol{\beta}}(\cdot, \cdot)$ is the semi-norm induced by the bilinear form $\mathrm{s}_{\boldsymbol{\beta}}$ defined by (3.10).

Proposition 4.6 (Coercivity). Assume that (H1) holds. Then,

$$
\frac{1}{2}\|\mathrm{v}\|^{2} \leq \mathbb{A}_{\boldsymbol{\beta}, \boldsymbol{\mu}}(\mathrm{v}, \mathrm{v}), \quad \forall \mathrm{v} \in \mathcal{E} .
$$

Consequently, the discrete problem (3.14) is well-posed. 
TABLE 1. Stability of the discrete problem (3.14) with respect to $\lambda_{b}$ and the mesh-size $h$.

\begin{tabular}{c|cc}
\hline$\lambda_{b}>0$ & $-\frac{1}{2 \vartheta \tau}<\lambda_{b} \leq 0$ \\
\hline$($ H1 $)$ & $($ H2 $)$ \\
\hline & $\boldsymbol{\mu}=-\nabla \boldsymbol{\beta}^{\mathrm{T}}$ & $\boldsymbol{\mu} \neq-\nabla \boldsymbol{\beta}^{\mathrm{T}}$ \\
\hline$h \in \mathbb{R}_{>0}$ & $h \in \mathbb{R}_{>0}$ & $h \in\left(0, h_{0}\left(1+2 \vartheta \tau \lambda_{b}\right)\right)$ \\
\hline
\end{tabular}

\subsection{Well-posedness under (H2)}

In this section, we address the stability of the bilinear form $\mathbb{A}_{\boldsymbol{\beta}, \boldsymbol{\mu}}$ under the hypothesis $(\mathbf{H 2})$. We consider the reference length $h_{0}^{-1}=4 C_{\sharp}^{2} L_{\zeta} \tau\left\|\boldsymbol{\mu}+\nabla \boldsymbol{\beta}^{\mathrm{T}}\right\|_{L^{\infty}(\Omega)}$, where $C_{\sharp}$ results from Proposition 4.1 and $L_{\zeta}=|\zeta|_{W^{1, \infty}(\Omega)}$ is the Lipschitz constant of $\zeta$. If $\boldsymbol{\mu}=-\nabla \boldsymbol{\beta}^{\mathrm{T}}$, we conventionally set $h_{0}=+\infty$. Recalling that $\lambda_{b}$ denotes the smallest eigenvalue of the tensor $\boldsymbol{\sigma}_{\boldsymbol{\beta}, \boldsymbol{\mu}}$ over the domain $\Omega$, we assume that

$$
1+2 \vartheta \tau \lambda_{b}>0 \text { and } h<h_{0}\left(1+2 \vartheta \tau \lambda_{b}\right),
$$

where $\vartheta>0$ is a non-dimensional constant that linearly depends on $\|\zeta\|_{L^{\infty}(\Omega)}+C_{\mathrm{T}} C_{\sharp} L_{\zeta} \max \left(|\Omega|^{\frac{1}{d}},\|\boldsymbol{\beta}\|_{L^{\infty}(\Omega)} \tau\right)$. By convention, the second condition in (4.6) is void if $\boldsymbol{\mu}=-\nabla \boldsymbol{\beta}^{\mathrm{T}}$.

Proposition 4.7 (Inf-sup stability). Assume that (H2) and (4.6) hold. Then, there exists $\varrho>0$ such that

$$
\varrho\|\mathrm{v}\| \leq \sup _{\mathrm{w} \in \mathcal{E},\|\mathrm{w}\|=1} \mathbb{A}_{\boldsymbol{\beta}, \boldsymbol{\mu}}(\mathrm{v}, \mathrm{w}), \quad \forall \mathrm{v} \in \mathcal{E} .
$$

Consequently, the discrete problem (3.14) is well-posed.

In the proof of Proposition 4.7, the idea is to introduce a discrete test function $\zeta \vee \in \mathcal{E}$ defined as $(\zeta \mathrm{v})_{e}=\zeta\left(\boldsymbol{x}_{e}\right) \mathrm{v}_{e}$ for all $\mathrm{v} \in \mathcal{E}$ and for all $e \in \mathrm{E}$. The key argument to obtain the well-posedness of the discrete problem (3.14) under hypothesis (H2) is then to bound the commutator $\boldsymbol{\delta}$ defined as

$$
\boldsymbol{\delta}(\mathrm{v})_{\mid c}=\mathbf{L}_{\mathcal{E}_{c}}(\zeta \mathrm{v})-\zeta \mathbf{L}_{\mathcal{E}_{c}}(\mathrm{v}), \quad \forall \mathrm{v} \in \mathcal{E}_{c}, \quad \forall c \in \mathrm{C} .
$$

Lemma 4.8 (Bounds on $\boldsymbol{\delta}$ ). For all $c \in \mathrm{C}$, we have

$$
\|\boldsymbol{\delta}(\mathrm{v})\|_{\boldsymbol{L}^{2}(c)} \leq 2 C_{\sharp} L_{\zeta} h_{c}\|\mathrm{v}\|_{2, c}, \quad \forall \mathrm{v} \in \mathcal{E}_{c} .
$$

and for all $f \in \mathrm{F}_{c}$,

$$
\|\boldsymbol{\delta}(\mathrm{v})\|_{\boldsymbol{L}^{2}(f)} \leq 2 C_{T} C_{\sharp} L_{\zeta} h_{c}^{\frac{1}{2}}\|\mathrm{v}\|_{2, c}, \quad \forall \mathrm{v} \in \mathcal{E}_{c} .
$$

Table 1 recapitulates the different situations where the discrete problem (3.14) is well-posed.

\subsection{Bound on consistency error and a priori error estimate}

In this section, we derive an a priori error estimate by bounding the consistency error

$$
\mathbb{E}(\boldsymbol{u})=\sup _{\mathrm{v} \in \mathcal{E},\|v\|=1}\left|\mathbb{A}_{\boldsymbol{\beta}, \boldsymbol{\mu}}\left(\mathrm{R}_{\mathcal{E}}(\boldsymbol{u}), \mathrm{v}\right)-\Sigma\left(\boldsymbol{s}, \boldsymbol{u}_{D} ; \mathrm{v}\right)\right| .
$$

In what follows, the notation $A \lesssim B$ stands for $A \leq C B$ where $C$ is a positive constant uniform with respect to the mesh size and the model parameters. 

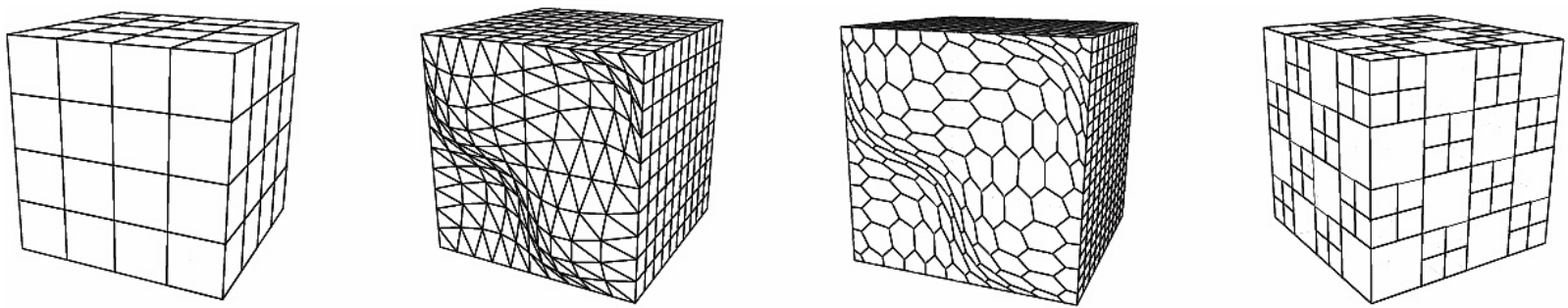

FiguRE 4. Examples of meshes from the four sequences. From left to right: hexahedral mesh $(\mathrm{H})$, prismatic mesh with triangular basis $(\operatorname{PrT})$, prismatic mesh with hexagonal basis ( $\operatorname{PrG})$, and Checkerboard mesh with non-matching interfaces (CB).

Lemma 4.9 (Bound on consistency error). Assume that the exact solution satisfies $\boldsymbol{u} \in \boldsymbol{W}^{1, q}(\Omega)$ with $q \in[1,2]$. Then, the following holds:

$$
\begin{aligned}
\mathbb{E}(\boldsymbol{u}) \lesssim & \left(\sum_{c \in \mathrm{C}}\left\|\nabla \boldsymbol{\beta}+\boldsymbol{\mu}^{\mathrm{T}}-(\nabla \cdot \boldsymbol{\beta}) \boldsymbol{I} \boldsymbol{d}\right\|_{\boldsymbol{L}^{\infty}(c)}^{q} \tau^{\frac{q}{2}} h_{c}^{\frac{d}{2}(q-2)}\left\|\boldsymbol{u}-\mathcal{I}_{\mathcal{E}_{c}}(\boldsymbol{u})\right\|_{\boldsymbol{L}^{q}(c)}^{q}\right)^{\frac{1}{q}} \\
& +\left(\sum_{c \in \mathrm{C}} \sum_{\mathfrak{p} \in \mathfrak{P}_{c}}\|\boldsymbol{\beta}\|_{\boldsymbol{L}^{\infty}(c)}^{\frac{q}{2}} h_{c}^{\frac{(d-1)}{2}(q-2)}\left\|\boldsymbol{u}-\mathcal{I}_{\mathcal{E}_{c}}(\boldsymbol{u})\right\|_{\boldsymbol{L}^{q}(\partial \mathfrak{p})}^{q}\right)
\end{aligned}
$$

We can now state the main result of this paper which follows from Lemmata 4.5 and 4.9.

Theorem 4.10 (A priori estimate). Assume that the assumptions stated in Table 1 hold. Assume that the exact solution of (1.1) satisfies $\boldsymbol{u} \in \boldsymbol{W}^{1, q}(\Omega)$ with $q \in\left(\frac{2 d}{d+1}, 2\right]$. Then, we have

$$
\begin{aligned}
\left\|\mathrm{u}-\mathrm{R}_{\mathcal{E}}(\boldsymbol{u})\right\| \lesssim & \left(\sum_{c \in \mathrm{C}}\left\|\nabla \boldsymbol{\beta}+\boldsymbol{\mu}^{\mathrm{T}}-(\nabla \cdot \boldsymbol{\beta}) \boldsymbol{I} \boldsymbol{d}\right\|_{\boldsymbol{L}^{\infty}(c)}^{q} \tau^{\frac{q}{2}} h_{c}^{\frac{d+2}{2}\left(q-\frac{2 d}{d+2}\right)}|\boldsymbol{u}|_{\boldsymbol{W}^{1, q}(\hat{c})}^{q}\right)^{\frac{1}{q}} \\
& +\left(\sum_{c \in \mathrm{C}} \sum_{\mathfrak{p} \in \mathfrak{P}_{c}}\|\boldsymbol{\beta}\|_{\boldsymbol{L}^{\infty}(c)}^{\frac{q}{2}} h_{c}^{\frac{d+1}{2}\left(q-\frac{2 d}{d+1}\right)}|\boldsymbol{u}|_{\boldsymbol{W}^{1, q}(\hat{c})}^{q}\right)^{\frac{1}{q}}
\end{aligned}
$$

For $d=3$, it follows that $\left\|\mathbf{u}-\mathrm{R}_{\mathcal{E}}(\boldsymbol{u})\right\|=\mathcal{O}\left(h^{2-\frac{3}{q}}\right)$ for all $q \in\left(\frac{3}{2}, 2\right]$.

\section{NumERicAl RESUlts}

We investigate numerically the edge-based scheme (3.14) on four sequences of three-dimensional polyhedral meshes. Each mesh is obtained as a uniform refinement of an initial mesh. Meshes from the first sequence, denoted $\mathrm{H}$, are composed of hexahedra, those from the second one, denoted PrT, are composed of prisms with a triangular basis, those from the third one, denoted PrG, are composed of prisms with a hexagonal basis, and those of the last one, denoted $\mathrm{CB}$, are composed of hexahedra with non-matching interfaces; see Figure 4. The domain is the unit cube $\Omega:=[0,1]^{3}$. The exact solution corresponds to a Taylor-Green velocity field, the advective vector field $\boldsymbol{\beta}$ is affine (see Fig. 5 , left panel) and the reaction tensor $\boldsymbol{\mu}$ is diagonal and constant:

$$
\boldsymbol{u}=\left(\begin{array}{c}
\sin (\pi x) \cos (\pi y / 2) \cos (\pi z / 2) \\
\cos (\pi x / 2) \sin (\pi y) \cos (\pi z / 2) \\
\cos (\pi x / 2) \cos (\pi y / 2) \sin (\pi z)
\end{array}\right), \quad \boldsymbol{\beta}=\frac{1}{2}\left(\begin{array}{c}
(x-2 y) / 2 \\
(y-2 x) / 2 \\
-z
\end{array}\right), \quad \boldsymbol{\mu}=\frac{1}{2} \mathbf{I} \mathbf{d}
$$



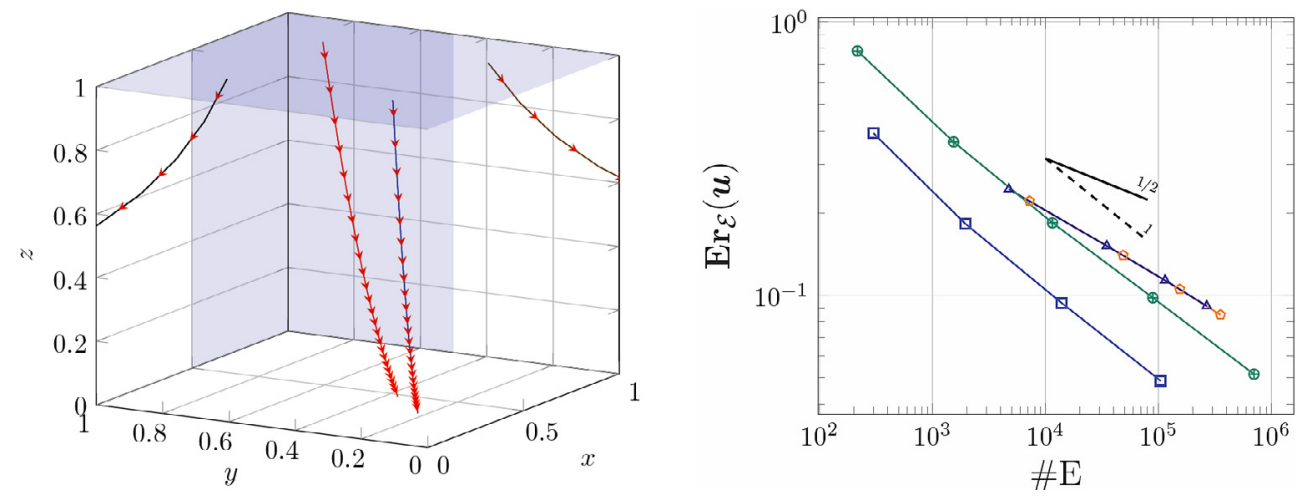

FiguRE 5. Left panel: Inflow boundary $\partial \Omega^{-}$in blue and some streamlines of the vector field $\boldsymbol{\beta}$. Right panel: Discrete errors on H $(\neg-)$, PrT $(\triangleleft)$, PrG $(\neg)$, and CB $(\neg-)$ mesh sequences. (Color online)

\begin{tabular}{ccccc}
$\# \mathrm{E}$ & $\mathbf{S t}$ & $\operatorname{Er}_{\mathcal{E}}(\boldsymbol{u})$ & $\frac{\# \mathrm{E}}{\# \mathrm{~V}}$ & $\frac{\# \mathrm{E}}{\# \mathrm{C}}$ \\
\hline $3.0 \mathrm{e}+02$ & 21 & $3.9 \mathrm{e}-01$ & 2.40 & 4.69 \\
$1.9 \mathrm{e}+03$ & 25 & $1.8 \mathrm{e}-01$ & 2.67 & 3.80 \\
$1.4 \mathrm{e}+04$ & 28 & $9.4 \mathrm{e}-02$ & 2.82 & 3.39 \\
$1.0 \mathrm{e}+05$ & 30 & $4.9 \mathrm{e}-02$ & 2.91 & 3.19
\end{tabular}

\begin{tabular}{ccccc}
$\# \mathrm{E}$ & $\mathbf{S t}$ & $\operatorname{Er}_{\mathcal{E}}(\boldsymbol{u})$ & $\frac{\# \mathrm{E}}{\# \mathrm{~V}}$ & $\frac{\# \mathrm{E}}{\# \mathrm{C}}$ \\
\hline $4.7 \mathrm{e}+03$ & 38 & $2.4 \mathrm{e}-01$ & 3.55 & 2.37 \\
$3.5 \mathrm{e}+04$ & 46 & $1.5 \mathrm{e}-01$ & 3.76 & 2.18 \\
$1.1 \mathrm{e}+05$ & 48 & $1.1 \mathrm{e}-01$ & 3.84 & 2.12 \\
$2.7 \mathrm{e}+05$ & 49 & $9.1 \mathrm{e}-02$ & 3.88 & 2.09
\end{tabular}

\begin{tabular}{ccccc}
$\# \mathrm{E}$ & $\mathbf{S t}$ & $\operatorname{Er}_{\mathcal{E}}(\boldsymbol{u})$ & $\frac{\# \mathrm{E}}{\# \mathrm{~V}}$ & $\frac{\# \mathrm{E}}{\# \mathrm{C}}$ \\
\hline $7.2 \mathrm{e}+03$ & 83 & $2.2 \mathrm{e}-01$ & 2.34 & 5.95 \\
$4.9 \mathrm{e}+04$ & 110 & $1.4 \mathrm{e}-01$ & 2.41 & 5.51 \\
$1.5 \mathrm{e}+05$ & 120 & $1.1 \mathrm{e}-01$ & 2.44 & 5.35 \\
$3.5 \mathrm{e}+05$ & 125 & $8.5 \mathrm{e}-02$ & 2.45 & 5.26
\end{tabular}

\begin{tabular}{ccccc}
$\# \mathrm{E}$ & $\mathrm{St}$ & $\operatorname{Er}_{\mathcal{E}}(\boldsymbol{u})$ & $\frac{\# \mathrm{E}}{\# \mathrm{~V}}$ & $\frac{\# \mathrm{E}}{\# \mathrm{C}}$ \\
\hline $1.5 \mathrm{e}+03$ & 112 & $3.6 \mathrm{e}-01$ & 2.46 & 5.33 \\
$1.2 \mathrm{e}+04$ & 144 & $1.8 \mathrm{e}-01$ & 2.61 & 5.00 \\
$8.9 \mathrm{e}+04$ & 162 & $9.8 \mathrm{e}-02$ & 2.70 & 4.83 \\
$7.0 \mathrm{e}+05$ & 180 & $5.1 \mathrm{e}-02$ & 2.75 & 4.75
\end{tabular}

FiguRE 6. Mean stencil St and discrete error $\operatorname{Er}_{\mathcal{E}}(\boldsymbol{u})$ for the $\mathrm{H}$ (upper left panel), PrT (upper right panel), $\operatorname{PrG}$ (lower left panel), and the $\mathrm{CB}$ (lower right panel) mesh sequences.

Note that $\nabla \cdot \boldsymbol{\beta}=0$ and that the eigenvalues of the tensor $\boldsymbol{\sigma}_{\boldsymbol{\beta}, \boldsymbol{\mu}}$ are $\left\{0, \frac{1}{2}, \frac{5}{2}\right\}$, so that the discrete scheme (3.14) is well-posed owing to Proposition 4.7 if the mesh size is small enough.

We perform a convergence study by computing the relative discrete $\boldsymbol{L}^{2}$-error attached to edge dofs, denoted $\operatorname{Er}_{\mathcal{E}}(\boldsymbol{u})$, and defined by

$$
\operatorname{Er}_{\mathcal{E}}(\boldsymbol{u})=\frac{\left\|\mathrm{u}-\mathrm{R}_{\mathcal{E}}(\boldsymbol{u})\right\|_{2}}{\left\|\mathrm{R}_{\mathcal{E}}(\boldsymbol{u})\right\|_{2}}
$$

with the norm $\|\cdot\|_{2}$ on every cell of the mesh by (4.1). The convergence rates, shown in the right panel of Figure 5, lie between $\frac{1}{2}$ and 1 for the PrT and PrG mesh sequences and are closer to 1 for the $\mathrm{H}$ and CB mesh sequences. Note that the considered meshes being quasi-uniform, we have $h \sim(\# \mathrm{E})^{-1 / 3}$; the reference slopes indicated in Figure 5 are based on this scaling, i.e., are with respect to $h$. Table 6 provides additional information on the computational costs by reporting the size of the linear system (\#E), the mean stencil St, the values of the discrete error $\operatorname{Er}_{\mathcal{E}}(\boldsymbol{u})$, and the ratios $\# \mathrm{E} / \# \mathrm{~V}$ and $\# \mathrm{E} / \# \mathrm{C}$, indicating that the present scheme may involve less dofs than traditional Finite Volume schemes placing $\mathbb{R}^{d}$-valued unknowns at mesh vertices or at mesh cells. Note that owing to the Euler-Poincaré characteristic formula (in dimension $d=3$; (see e.g. [26], Chap. 8)), $\frac{\# \mathrm{~V}}{\# \mathrm{E}}+\frac{\# \mathrm{~F}}{\# \mathrm{E}}-\frac{\# \mathrm{C}}{\# \mathrm{E}}=\frac{2}{\# \mathrm{E}}-1 \approx 1$. 
Remark 5.1 (Stabilization parameter). As observed in Bonelle et al. [4], one can reformulate the basis functions $\ell_{e, c}$ as a consistent term plus a stabilization term:

$$
\left.\boldsymbol{\ell}_{e, c}\right|_{\mathfrak{p}_{e^{\prime}, c}}=\underbrace{\frac{\tilde{\boldsymbol{f}}_{c}(e)}{|c|}}_{\text {Consistent term }}+\frac{1}{d} \underbrace{\left(\frac{\tilde{\boldsymbol{f}}_{c}(e)}{\left|\mathfrak{p}_{e, c}\right|} \delta_{e, e^{\prime}}-\frac{\boldsymbol{e}^{\prime} \cdot \tilde{\boldsymbol{f}}_{c}(e)}{\left|\mathfrak{p}_{e^{\prime}, c}\right|} \frac{\tilde{\boldsymbol{f}}_{c}\left(e^{\prime}\right)}{|c|}\right)}_{\text {Stabilization term }}, \quad \forall e^{\prime} \in \mathrm{E}_{c} .
$$

Numerical experiments show that it is possible to replace the parameter $d^{-1}$ by a positive value that is reasonably close to $d^{-1}$; however, in the stability analysis, this modification impacts the property $(\ell 1)$ which is used to obtain the lower bound in Proposition 4.1.

\section{Proofs}

\subsection{Properties of the reconstruction map}

Proof of Proposition 4.1. Let $c \in \mathrm{C}$, let $\mathrm{v} \in \mathcal{E}_{c}$ and let $q \in[1, \infty)$.

(i) Lower bound. Owing to the definition (3.1) of $\mathbf{L}_{\mathcal{E}_{c}}$, we have for all $e \in \mathrm{E}_{c},\left.\mathbf{L}_{\mathcal{E}_{c}}(\mathrm{v})\right|_{\mid \mathfrak{p}_{e, c}}=\mathrm{v}_{e} \boldsymbol{a}_{e}+\boldsymbol{b}_{e}$ with

$$
\boldsymbol{a}_{e}=\frac{\boldsymbol{e}}{|e|^{2}} \text { and } \boldsymbol{b}_{e}=\left(\ell_{e, c}-\frac{e}{|e|^{2}}\right) \mathrm{v}_{e}+\sum_{e^{\prime} \in \mathrm{E}_{c} \backslash\{e\}} \mathrm{v}_{e^{\prime}} \ell_{e^{\prime}, c^{\prime}} \text {. }
$$

Recalling that $\|\cdot\|_{L^{q}(c)}=\left\||\cdot|_{\ell^{2}}\right\|_{L^{q}}$, we infer that

$$
\left\|\mathbf{L}_{\mathcal{E}_{c}}(\mathrm{v})\right\|_{L^{q}(c)}^{q}=\sum_{e \in \mathrm{E}_{c}}\left\|\left|\mathrm{v}_{e} \boldsymbol{a}_{e}+\boldsymbol{b}_{e}\right|_{\ell^{2}}\right\|_{L^{q}\left(\mathfrak{p}_{e, c}\right)}^{q} .
$$

Using the Property $(\boldsymbol{\ell} 1)$, we observe that $\boldsymbol{a}_{e} \cdot \boldsymbol{b}_{e} \equiv 0$ on $\mathfrak{p}_{e, c}$, so that $\left|v_{e} \boldsymbol{a}_{e}+\boldsymbol{b}_{e}\right|_{\ell^{2}} \geq\left|v_{e} \boldsymbol{a}_{e}\right|_{\ell^{2}}$, whence

$$
\left\|\mathbf{L}_{\mathcal{E}_{c}}(\mathrm{v})\right\|_{L^{q}(c)}^{q} \geq \sum_{e \in \mathrm{E}_{c}}\left\|\left|v_{e} \boldsymbol{a}_{e}\right|_{\ell^{2}}\right\|_{L^{q}\left(\mathfrak{p}_{e, c}\right)}^{q}=\sum_{e \in \mathrm{E}_{c}}\left|\mathrm{v}_{e}\right|^{q}\left\|\boldsymbol{a}_{e}\right\|_{\boldsymbol{L}^{q}\left(\mathfrak{p}_{e, c}\right)}^{q} .
$$

Hence, the expected lower bound follows from $\left\|\boldsymbol{a}_{e}\right\|_{\boldsymbol{L}^{q}\left(\mathfrak{p}_{e, c}\right)}^{q}=\frac{\left|\mathfrak{p}_{e, c}\right|}{|e|^{q}}$.

(ii) Upper bound. The discrete Hölder inequality yields

$$
\left\|\mathbf{L}_{\mathcal{E}_{c}}(\mathrm{v})\right\|_{\mathbf{L}^{q}(c)}^{q} \leq\left(\# \mathrm{E}_{c}\right)^{q-1} \sum_{e \in \mathrm{E}_{c}}\left|\mathrm{v}_{e}\right|^{q}\left\|\boldsymbol{\ell}_{e, c}\right\|_{\boldsymbol{L}^{q}(c)}^{q} .
$$

Since $\left\|\boldsymbol{\ell}_{e, c}\right\|_{\boldsymbol{L}^{q}(c)}^{q} \leq|c|\left\|\boldsymbol{\ell}_{e, c}\right\|_{\boldsymbol{L}^{\infty}(c)}^{q}$, we have $\left\|\boldsymbol{\ell}_{e, c}\right\|_{\boldsymbol{L}^{q}(c)}^{q} \leq C_{\sharp}^{q}\left(\# \mathrm{E}_{c}\right)^{1-q} \frac{\left|\mathfrak{p}_{e, c}\right|}{|e|^{q}}$ with the constant

$$
C_{\sharp}=\left(\# \mathrm{E}_{c}\right)^{1-\frac{1}{q}} \max _{e \in \mathrm{E}_{c}}\left(\left(\frac{|c|}{\left|\mathfrak{p}_{e, c}\right|}\right)^{\frac{1}{q}}|e|\left\|\boldsymbol{\ell}_{e, c}\right\|_{\boldsymbol{L}^{\infty}(c)}\right),
$$

that is uniformly bounded owing to mesh regularity, yielding the expected upper bound. Specifically, a straightforward calculation shows that

$$
\left.\left|\boldsymbol{\ell}_{e, c}\right|_{\mathfrak{p}_{e, c}}\right|_{\ell^{2}} \leq \frac{\left|\tilde{f}_{c}(e)\right|}{|c|}\left(\frac{|c|}{d\left|\mathfrak{p}_{e, c}\right|}\right) \quad \text { and }\left.\quad\left|\boldsymbol{\ell}_{e, c}\right|_{\mathfrak{p}^{\prime}, c}\right|_{\ell^{2}} \leq \frac{\left|\tilde{f}_{c}(e)\right|}{|c|}\left(1+\frac{1}{\cos ^{2}\left(\boldsymbol{t}_{e^{\prime}}, \boldsymbol{n}_{\tilde{f}_{c}\left(e^{\prime}\right)}\right)}\right)^{\frac{1}{2}}
$$

leading to

$$
|e|\left\|\boldsymbol{\ell}_{e, c}\right\|_{\boldsymbol{L}^{\infty}(c)} \leq\left(\frac{|e|\left|\tilde{f}_{c}(e)\right|}{|c|}\right) \max \left\{\left(\frac{|c|}{d\left|\mathfrak{p}_{e, c}\right|}\right), \max _{e^{\prime} \in \mathrm{E}_{c}, e^{\prime} \neq e}\left(1+\frac{1}{\cos ^{2}\left(\boldsymbol{t}_{e^{\prime}}, \boldsymbol{n}_{\tilde{f}_{c}\left(e^{\prime}\right)}\right)}\right)^{\frac{1}{2}}\right\} .
$$


Proof of Proposition 4.4. Let $c \in \mathrm{C}$ and let $e^{\prime} \in \mathrm{E}_{c}$. The consistency property relies on the property $(\ell 2)$. Indeed, given $\boldsymbol{U} \in \mathbb{P}_{0}\left(\hat{c} ; \mathbb{R}^{d}\right)$, we infer that, for all $\boldsymbol{x} \in \mathfrak{p}_{e^{\prime}, c}$,

$$
\mathrm{L}_{\mathcal{E}_{c}} \mathrm{R}_{\mathcal{E}_{c}}(\boldsymbol{U})(\boldsymbol{x})=\sum_{e \in \mathrm{E}_{c}} \mathrm{R}_{\mathcal{E}_{c}}(\boldsymbol{U})_{\mid e} \boldsymbol{\ell}_{e, c}(\boldsymbol{x})=\sum_{e \in \mathrm{E}_{c}}(\boldsymbol{U} \cdot \boldsymbol{e}) \boldsymbol{\ell}_{e, c}(\boldsymbol{x})=\left(\sum_{e \in \mathrm{E}_{c}} \boldsymbol{\ell}_{e, c}(\boldsymbol{x}) \otimes \boldsymbol{e}\right) \boldsymbol{U}=\boldsymbol{U} .
$$

Proof of Lemma 4.5. Let $c \in \mathrm{C}$ and let $\boldsymbol{v} \in \boldsymbol{W}^{1, q}(\hat{c})$ with $q \in[1, \infty)$. Owing to the triangle inequality and the $\mathbb{P}_{0}$-consistency of the reconstruction map from Proposition 4.4, we infer that

$$
\left\|\boldsymbol{v}-\mathcal{I}_{\mathcal{E}_{c}}(\boldsymbol{v})\right\|_{\boldsymbol{L}^{q}(c)} \leq\left\|\boldsymbol{v}-\boldsymbol{v}_{\hat{c}}\right\|_{\boldsymbol{L}^{q}(c)}+\left\|\mathcal{I}_{\mathcal{E}_{c}}\left(\boldsymbol{v}-\boldsymbol{v}_{\hat{c}}\right)\right\|_{\boldsymbol{L}^{q}(c)}
$$

with $\boldsymbol{v}_{\hat{c}}=|\hat{c}|^{-1} \int_{\hat{c}} \boldsymbol{v}$. In addition, we observe that, for all $\boldsymbol{w} \in L^{q}(\hat{c})$,

$$
\left\|\mathrm{R}_{\mathcal{E}_{c}}(\boldsymbol{w})\right\|_{q, c}^{q}=\sum_{e \in \mathrm{E}_{c}} \frac{\left|\mathfrak{p}_{e, c}\right|}{|e|^{q}}\left|\frac{1}{\left|\mathfrak{p}_{e}\right|} \int_{\mathfrak{p}_{e}} \boldsymbol{w} \cdot \boldsymbol{e}\right|^{q} \leq \sum_{e \in \mathrm{E}_{c}} \frac{\left|\mathfrak{p}_{e, c}\right|}{\left|\mathfrak{p}_{e}\right|^{q}}\|\boldsymbol{w}\|_{L^{1}\left(\mathfrak{p}_{e}\right)}^{q} \leq \sum_{e \in \mathrm{E}_{c}} \frac{1}{\left|\mathfrak{p}_{e}\right|^{q-1}}\|\boldsymbol{w}\|_{\boldsymbol{L}^{1}\left(\mathfrak{p}_{e}\right)}^{q},
$$

where we have used that $\left|\mathfrak{p}_{e, c}\right| \leq\left|\mathfrak{p}_{e}\right|$ to infer the last inequality. Owing to the Hölder inequality, it then follows that $\|\boldsymbol{w}\|_{\boldsymbol{L}^{1}\left(\mathfrak{p}_{e}\right)}^{q} \leq\|\boldsymbol{w}\|_{\boldsymbol{L}^{q}\left(\mathfrak{p}_{e}\right)}^{q}\|1\|_{L^{q^{\prime}}\left(\mathfrak{p}_{e}\right)}^{q}$ with $\frac{1}{q}+\frac{1}{q^{\prime}}=1$. Since $\|1\|_{L^{q^{\prime}}\left(\mathfrak{p}_{e}\right)}^{q}=\left|\mathfrak{p}_{e}\right|^{q-1}$, we infer that

$$
\left\|\mathrm{R}_{\mathcal{E}_{c}}(\boldsymbol{w})\right\|_{q, c}^{q} \leq\|\boldsymbol{w}\|_{L^{q}(\hat{c})}^{q} .
$$

Using this estimate and the upper bound from Proposition 4.1, we obtain

$$
\left\|\mathcal{I}_{\mathcal{E}_{c}}\left(\boldsymbol{v}-\boldsymbol{v}_{\hat{c}}\right)\right\|_{\boldsymbol{L}^{q}(c)} \leq C_{\sharp}\left\|\mathrm{R}_{\mathcal{E}_{c}}\left(\boldsymbol{v}-\boldsymbol{v}_{\hat{c}}\right)\right\|_{q, c} \leq C_{\sharp}\left\|\boldsymbol{v}-\boldsymbol{v}_{\hat{c}}\right\|_{\boldsymbol{L}^{q}(c)},
$$

so that $\left\|\boldsymbol{v}-\mathcal{I}_{\mathcal{E}_{c}}(\boldsymbol{v})\right\|_{\boldsymbol{L}^{q}(c)} \leq\left(1+C_{\sharp}\right)\left\|\boldsymbol{v}-\boldsymbol{v}_{\hat{c}}\right\|_{\boldsymbol{L}^{q}(c)}$. Hence, $\left\|\boldsymbol{v}-\mathcal{I}_{\mathcal{E}_{c}}(\boldsymbol{v})\right\|_{\boldsymbol{L}^{q}(c)} \leq\left(1+C_{\sharp}\right) \phi_{\hat{c}, q} h_{c}|\boldsymbol{v}|_{\boldsymbol{W}^{1, q}(\hat{c})}$ with

$$
\phi_{\hat{c}, q}=\sup _{\boldsymbol{w} \in \boldsymbol{W}^{1, q}(\hat{c})} \frac{\left\|\boldsymbol{w}-\boldsymbol{w}_{\hat{c}}\right\|_{\boldsymbol{L}^{q}(\hat{c})}}{h_{c}|\boldsymbol{w}|_{\boldsymbol{W}^{1, q}(\hat{c})}}
$$

Finally, we observe that the diamond $\hat{c}$ can be decomposed as

$$
\hat{c}=\bigcup_{e \in \mathrm{E}_{c}} \mathfrak{p}_{e}=\bigcup_{e \in \mathrm{E}_{c}} \bigcup_{c \in \mathrm{C}_{e}} \mathfrak{p}_{e, c}
$$

where $\mathfrak{p}_{e, c}$ consists of two tetrahedra, so that $\hat{c}$ is composed of $2 \sum_{e \in \mathrm{E}_{c}} \# \mathrm{C}_{e}$ tetrahedra connected through elements of $\mathfrak{F}_{c}$ and $\mathfrak{F}_{f}$ with $f \in \mathrm{F}_{c}$. Then, proceeding as Ern and Guermond in ([15], Lem. 5.7), we infer that the quantity $\phi_{\hat{c}, q}$ is uniformly bounded for all $c \in \mathrm{C}$ and all $q \in[1, \infty)$.

\subsection{Well-posedness under (H1)}

Proof of Proposition 4.6. Let $c \in \mathrm{C}$ and consider $\mathrm{v}, \mathrm{w} \in \mathcal{E}_{c}$. The definition of the bilinear form $\mathrm{g}_{\boldsymbol{\beta}, \boldsymbol{\mu} ; c}$ together with the definition of the tensor $\boldsymbol{\sigma}_{\boldsymbol{\beta}, \boldsymbol{\mu}}$ yield

$$
\mathrm{g}_{\boldsymbol{\beta}, \boldsymbol{\mu} ; c}(\mathrm{v}, \mathrm{w})+\mathrm{g}_{\boldsymbol{\beta}, \boldsymbol{\mu} ; c}(\mathrm{w}, \mathrm{v})=\int_{c} \mathbf{L}_{\mathcal{E}_{c}}(\mathrm{v}) \cdot \boldsymbol{\sigma}_{\boldsymbol{\beta}, \boldsymbol{\mu}} \cdot \mathbf{L}_{\mathcal{E}_{c}}(\mathrm{w})+\sum_{\mathfrak{p} \in \mathfrak{P}_{c}} \int_{\mathfrak{p}} \nabla \cdot\left(\boldsymbol{\beta} \mathbf{L}_{\mathcal{E}_{c}}(\mathrm{v}) \cdot \mathbf{L}_{\mathcal{E}_{c}}(\mathrm{w})\right) .
$$

Choosing $\mathrm{w}=\mathrm{v}$ in this relation leads to

$$
\frac{1}{2} \sum_{\mathfrak{p} \in \mathfrak{P}_{c}} \int_{\mathfrak{p}} \nabla \cdot\left(\boldsymbol{\beta}\left|\mathbf{L}_{\mathcal{E}_{c}}(\mathrm{v})\right|_{\ell^{2}}^{2}\right)-\sum_{\mathfrak{f} \in \mathfrak{F}_{c}} \int_{\mathfrak{f}}\left(\boldsymbol{\beta} \cdot \boldsymbol{n}_{\mathfrak{f}}\right) \llbracket \mathbf{L}_{\mathcal{E}}(\mathrm{v}) \rrbracket \cdot\left\{\left\{\mathbf{L}_{\mathcal{E}}(\mathrm{v})\right\}=\frac{1}{2} \sum_{f \in \mathrm{F}_{c}} \int_{f}\left(\boldsymbol{\beta} \cdot \boldsymbol{n}_{c}\right)\left|\mathbf{L}_{\mathcal{E}_{c}}(\mathrm{v})\right|_{\ell^{2}}^{2},\right.
$$


with $\boldsymbol{n}_{c}$ the unit outward normal vector to $c$, so that recalling the definition (3.9) of $\mathrm{n}_{\boldsymbol{\beta} ; c}$, we infer that

$$
\sum_{c \in \mathrm{C}}\left(\mathrm{g}_{\boldsymbol{\beta}, \boldsymbol{\mu} ; c}(\mathrm{v}, \mathrm{v})+\mathrm{n}_{\boldsymbol{\beta} ; c}(\mathrm{v}, \mathrm{v})\right)=\frac{1}{2} \sum_{c \in \mathrm{C}} \int_{c} \mathbf{L}_{\mathcal{E}_{c}}(\mathrm{v}) \cdot \boldsymbol{\sigma}_{\boldsymbol{\beta}, \boldsymbol{\mu}} \cdot \mathbf{L}_{\mathcal{E}_{c}}(\mathrm{v})+\frac{1}{2} \sum_{c \in \mathrm{C}} \sum_{f \in \mathrm{F}_{c}} \int_{f}\left(\boldsymbol{\beta} \cdot \boldsymbol{n}_{c}\right)\left|\mathbf{L}_{\mathcal{E}_{c}}(\mathrm{v})\right|_{\ell^{2}}^{2} .
$$

The above rightmost term is reformulated as

$$
\begin{aligned}
\frac{1}{2} \sum_{c \in \mathrm{C}} \sum_{f \in \mathrm{F}_{c}} \int_{f}\left(\boldsymbol{\beta} \cdot \boldsymbol{n}_{c}\right)\left|\mathbf{L}_{\mathcal{E}}(\mathrm{v})\right|_{\ell^{2}}^{2} & =\frac{1}{2} \sum_{f \in \mathrm{F}^{\partial}} \int_{f}(\boldsymbol{\beta} \cdot \boldsymbol{n})\left|\mathbf{L}_{\mathcal{E}_{c_{f}}}(\mathrm{v})\right|_{\ell^{2}}^{2}+\sum_{f \in \mathrm{F}^{\circ}} \sum_{\mathfrak{f} \in \mathfrak{F}_{f}} \int_{\mathfrak{f}}\left(\boldsymbol{\beta} \cdot \boldsymbol{n}_{\mathfrak{f}}\right) \llbracket \mathbf{L}_{\mathcal{E}}(\mathrm{v}) \rrbracket \cdot\left\{\left\{\mathbf{L}_{\mathcal{E}}(\mathrm{v})\right\}\right\} \\
& =\frac{1}{2} \sum_{f \in \mathrm{F}^{\partial}} \int_{f}(\boldsymbol{\beta} \cdot \boldsymbol{n})\left|\mathbf{L}_{\mathcal{E}_{c_{f}}}(\mathrm{v})\right|_{\ell^{2}}^{2}+\sum_{f \in \mathrm{F}^{\circ}} \mathrm{n}_{\boldsymbol{\beta} ; f}(\mathrm{v}, \mathrm{v})
\end{aligned}
$$

so that, using the definition (3.8) of $\mathrm{n}_{\boldsymbol{\beta}}$, we arrive at

$$
\mathrm{g}_{\boldsymbol{\beta}, \boldsymbol{\mu}}(\mathrm{v}, \mathrm{v})+\mathrm{n}_{\boldsymbol{\beta}}(\mathrm{v}, \mathrm{v})=\frac{1}{2} \sum_{c \in \mathrm{C}} \int_{c} \mathrm{~L}_{\mathcal{E}_{c}}(\mathrm{v}) \cdot \boldsymbol{\sigma}_{\boldsymbol{\beta}, \boldsymbol{\mu}} \cdot \mathbf{L}_{\mathcal{E}_{c}}(\mathrm{v})+\frac{1}{2} \sum_{f \in \mathrm{F}^{\partial}} \int_{f}(\boldsymbol{\beta} \cdot \boldsymbol{n})\left|\mathbf{L}_{\mathcal{E}_{c_{f}}}(\mathrm{v})\right|_{\ell^{2}}^{2} .
$$

Recalling the definition (3.3) of $\mathbb{A}_{\boldsymbol{\beta}, \boldsymbol{\mu}}$ and combining the above relation with the bilinear forms $\mathrm{s}_{\boldsymbol{\beta}}$ and $\mathrm{A}_{(\boldsymbol{\beta} \cdot \boldsymbol{n})^{-}}^{\partial}$, defined by (3.10) and (3.12) respectively, we obtain

$$
\mathbb{A}_{\boldsymbol{\beta}, \boldsymbol{\mu}}(\mathrm{v}, \mathrm{v})=\frac{1}{2} \sum_{c \in \mathrm{C}} \int_{c} \mathbf{L}_{\mathcal{E}_{c}}(\mathrm{v}) \cdot \boldsymbol{\sigma}_{\boldsymbol{\beta}, \boldsymbol{\mu}} \cdot \mathbf{L}_{\mathcal{E}_{c}}(\mathrm{v})+\frac{1}{2} \mathrm{~A}_{|\boldsymbol{\beta} \cdot \boldsymbol{n}|}^{\partial}(\mathrm{v}, \mathrm{v})+\frac{1}{2} \mathrm{~s}_{\boldsymbol{\beta}}(\mathrm{v}, \mathrm{v}) .
$$

The expected result is inferred from $(\mathbf{H 1})$.

\subsection{Well-posedness under (H2)}

Proof of Lemma 4.8. Let $\vee \in \mathcal{E}$ and let $c \in \mathrm{C}$.

(i) Proof of (4.8a). Let $\zeta_{c}$ be the mean-value of $\zeta$ over $c$ given by $\zeta_{c}=|c|^{-1} \int_{c} \zeta$. Since $\mathbf{L}_{\mathcal{E}_{c}}\left(\zeta_{c} \mathrm{v}\right)=\zeta_{c} \mathbf{L}_{\mathcal{E}_{c}}(\mathrm{v})$ because $\zeta_{c}$ is constant, we have $\boldsymbol{\delta}(\mathrm{v})_{\mid c}=\left(\zeta-\zeta_{c}\right) \mathbf{L}_{\mathcal{E}_{c}}(\mathrm{v})-\mathbf{L}_{\mathcal{E}_{c}}\left(\left(\zeta-\zeta_{c}\right) \mathrm{v}\right)$, so that the triangle inequality, the Hölder inequality and the upper bound in Proposition 4.1 yield

$$
\begin{aligned}
\|\boldsymbol{\delta}(\mathrm{v})\|_{L^{2}(c)} & \leq\left\|\zeta-\zeta_{c}\right\|_{L^{\infty}(c)}\left\|\mathbf{L}_{\mathcal{E}_{c}}(\mathrm{v})\right\|_{L^{2}(c)}+\left\|\mathbf{L}_{\mathcal{E}_{c}}\left(\left(\zeta-\zeta_{c}\right) \mathrm{v}\right)\right\|_{L^{2}(c)} \\
& \leq C_{\sharp}\left\|\zeta-\zeta_{c}\right\|_{L^{\infty}(c)}\|\mathrm{v}\|_{2, c}+C_{\sharp}\left\|\left(\zeta-\zeta_{c}\right) \mathrm{v}\right\|_{2, c} \\
& \leq 2 C_{\sharp}\left\|\zeta-\zeta_{c}\right\|_{L^{\infty}(c)}\|\mathrm{v}\|_{2, c} .
\end{aligned}
$$

Observing that $\left\|\zeta-\zeta_{c}\right\|_{L^{\infty}(c)} \leq L_{\zeta} h_{c}$, the expected result follows.

(ii) Proof of (4.8b). Let $\mathfrak{p} \in \mathfrak{P}_{c}$ and let $\mathfrak{f} \subset \partial \mathfrak{p}$. Owing to the multiplicative trace inequality (2.1), we have

$$
\|\boldsymbol{\delta}(\mathrm{v})\|_{\boldsymbol{L}^{2}(\mathfrak{f})} \leq C_{T}\|\boldsymbol{\delta}(\mathrm{v})\|_{\boldsymbol{L}^{2}(\mathfrak{p})}^{\frac{1}{2}}\left(h_{c}^{-\frac{1}{2}}\|\boldsymbol{\delta}(\mathrm{v})\|_{\boldsymbol{L}^{2}(\mathfrak{p})}^{\frac{1}{2}}+|\boldsymbol{\delta}(\mathrm{v})|_{\boldsymbol{H}^{1}(\mathfrak{p})}^{\frac{1}{2}}\right)
$$

Observe that $|\boldsymbol{\delta}(\mathrm{v})|_{\boldsymbol{H}^{1}(\mathfrak{p})}=\left\||\nabla \boldsymbol{\delta}(\mathrm{v})|_{\ell^{2}}\right\|_{L^{2}(\mathfrak{p})}$ where $|\nabla \boldsymbol{\delta}(\mathrm{v})|_{\ell^{2}}^{2}=\sum_{i, j}^{d}\left|\partial_{j} \boldsymbol{\delta}(\mathrm{v})_{i}\right|^{2}$ in the Cartesian basis of $\mathbb{R}^{d}$ and where $\partial_{i}$ is the weak derivative in the direction $i$. Since $\mathbf{L}_{\mathcal{E}_{c}}(\mathrm{v})$ is piece-wise constant on $\mathfrak{P}_{c}$, it then follows that $|\nabla \boldsymbol{\delta}(\mathrm{v})|_{\ell^{2}}^{2}=\sum_{i, j}^{d}\left|\mathbf{L}_{\mathcal{E}_{c}}(\mathrm{v})_{i} \partial_{j} \zeta\right|^{2}=\left|\mathbf{L}_{\mathcal{E}_{c}}(\mathrm{v})\right|_{\ell^{2}}^{2}|\nabla \zeta|_{\ell^{2}}^{2}$. As a result, $|\boldsymbol{\delta}(\mathrm{v})|_{\boldsymbol{H}^{1}(\mathfrak{p})} \leq L_{\zeta}\left\|\mathbf{L}_{\mathcal{E}_{c}}(\mathrm{v})\right\|_{\boldsymbol{L}^{2}(\mathfrak{p})}$. Moreover, proceeding as in (i), we infer that $\|\boldsymbol{\delta}(\mathrm{v})\|_{L^{2}(\mathfrak{p})} \leq 2 L_{\zeta} h_{c}\left\|\mathbf{L}_{\mathcal{E}_{c}}(\mathrm{v})\right\|_{\boldsymbol{L}^{2}(\mathfrak{p})}$. Collecting these bounds, we infer that

$$
\|\boldsymbol{\delta}(\mathrm{v})\|_{\boldsymbol{L}^{2}(\mathfrak{f})} \leq 2 C_{T} L_{\zeta} h_{c}^{\frac{1}{2}}\left\|\mathbf{L}_{\mathcal{E}_{c}}(\mathrm{v})\right\|_{\boldsymbol{L}^{2}(\mathfrak{p})} .
$$

Then, summing over $\mathfrak{F}_{f}$ and using the upper bound of Proposition 4.1 yield the expected result. 
In what follows, we consider the non-dimensional number $\omega_{\zeta}=L_{\zeta} \max \left(|\Omega|^{\frac{1}{d}},\|\boldsymbol{\beta}\|_{L^{\infty}(\Omega)} \tau\right)$.

Lemma 6.1 (Multiplicative stability). There exists $C_{\zeta}>0$ independent of the mesh size and the model parameters such that

$$
\|\zeta \mathrm{v}\| \leq C_{\zeta}\left(\|\zeta\|_{L^{\infty}(\Omega)}+\omega_{\zeta}\right)\|\mathrm{v}\|, \quad \forall \mathrm{v} \in \mathcal{E} .
$$

Proof. Let $\mathrm{v} \in \mathcal{E}$ and let us rewrite $\|\zeta \mathrm{v}\|^{2}$ as

$$
\begin{aligned}
\|\zeta \mathrm{v}\|^{2} & =\sum_{c \in \mathrm{C}} \tau^{-1}\|\zeta \mathrm{v}\|_{2, c}^{2}+\sum_{c \in \mathrm{C}} \mathrm{s}_{\boldsymbol{\beta}, c}(\zeta \mathrm{v}, \zeta \mathrm{v})+\sum_{f \in \mathrm{F}^{\partial}} \mathrm{A}_{|\boldsymbol{\beta} \cdot \boldsymbol{n}| ; f}(\zeta \mathrm{v}, \zeta \mathrm{v})+\sum_{f \in \mathrm{F}^{\circ}} \mathrm{s}_{\boldsymbol{\beta}, f}(\zeta \mathrm{v}, \zeta \mathrm{v}) \\
& =T_{1}+T_{2}+T_{3}+T_{4} .
\end{aligned}
$$

We want to use the Lipschitz regularity of $\zeta$ to bound separately these terms by $\|\mathrm{v}\|^{2}$. We recall the notation $\zeta_{c}=|c|^{-1} \int_{c} \zeta$ from the proof of Lemma 4.8 .

(i) Bound on $T_{1}$. First, the triangle inequality implies that

$$
\frac{1}{2} T_{1} \leq \sum_{c \in \mathrm{C}} \tau^{-1}\left\|\zeta_{c} \mathrm{v}\right\|_{2, c}^{2}+\sum_{c \in \mathrm{C}} \tau^{-1}\left\|\left(\zeta-\zeta_{c}\right) \mathrm{v}\right\|_{2, c}^{2}=T_{1,1}+T_{1,2}
$$

Since $\left|\zeta_{c}\right| \leq\|\zeta\|_{L^{\infty}(c)}$, we infer that $T_{1,1} \leq \sum_{c \in \mathrm{C}} \tau^{-1}\|\zeta\|_{L^{\infty}(c)}^{2}\|\mathrm{v}\|_{2, c}^{2} \leq\|\zeta\|_{L^{\infty}(\Omega)}^{2}\|v\|^{2}$. The bound $T_{1,2}$ easily follows from the Lipschitz regularity of $\zeta$ since $T_{1,2} \leq \sum_{c \in \mathrm{C}} \tau^{-1} L_{\zeta}^{2} h_{c}^{2}\|\mathrm{v}\|_{2, c}^{2} \leq L_{\zeta}^{2} h_{c}^{2}\|\mathrm{v}\|^{2}$. Combining these two bounds with $h_{c} \leq|\Omega|^{\frac{1}{d}}$ and the definition of $\omega_{\zeta}$ yields

$$
T_{1} \leq 2\left(\omega_{\zeta}^{2}+\|\zeta\|_{L^{\infty}(\Omega)}^{2}\right)\|\mathrm{v}\|^{2} .
$$

(ii) Bound on $T_{2}$. Since the bilinear form $\mathbf{s}_{\boldsymbol{\beta} ; c}$ is symmetric and positive, we infer that

$$
\frac{1}{2} T_{2} \leq \sum_{c \in \mathrm{C}} \mathrm{s}_{\boldsymbol{\beta} ; c}\left(\zeta_{c} \mathrm{v}, \zeta_{c} \mathrm{v}\right)+\sum_{c \in \mathrm{C}} \mathrm{s}_{\boldsymbol{\beta} ; c}\left(\left(\zeta-\zeta_{c}\right) \mathrm{v},\left(\zeta-\zeta_{c}\right) \mathrm{v}\right)=T_{2,1}+T_{2,2},
$$

and we have directly that $T_{2,1} \leq \sum_{c \in C}\|\zeta\|_{L^{\infty}(c)}^{2} \mathbf{s}_{\boldsymbol{\beta}, c}(\mathrm{v}, \mathrm{v}) \leq\|\zeta\|_{L^{\infty}(\Omega)}^{2}\|\mathrm{v}\|^{2}$. To bound $T_{2,2}$, we use the multiplicative trace inequality $(2.1)$ and that $\mathbf{L}_{\mathcal{E}_{c}}$ is piece-wise constant to infer that

$\mathrm{s}_{\boldsymbol{\beta} ; c}\left(\left(\zeta-\zeta_{c}\right) \mathrm{v},\left(\zeta-\zeta_{c}\right) \mathrm{v}\right)=\sum_{\mathfrak{f} \in \mathfrak{F}_{c}} \int_{\mathfrak{f}}\left|\boldsymbol{\beta} \cdot \boldsymbol{n}_{\mathfrak{f}}\right|\left|\llbracket \mathbf{L}_{\mathcal{E}_{c}}\left(\left(\zeta-\zeta_{c}\right) \mathrm{v}\right)\left\|\left.\right|_{\ell^{2}} ^{2} \leq 2 C_{T}^{2}\right\| \boldsymbol{\beta}\left\|_{\boldsymbol{L}^{\infty}(c)} \sum_{\mathfrak{f} \in \mathfrak{F}_{c}} \sum_{\mathfrak{p} \in \mathfrak{P}_{c} \cap \mathfrak{P}_{\mathfrak{f}}} h_{c}^{-1}\right\| \mathbf{L}_{\mathcal{E}_{c}}\left(\left(\zeta-\zeta_{c}\right) \mathrm{v}\right) \|_{\boldsymbol{L}^{2}(\mathfrak{p})}^{2}\right.$,

where $\mathfrak{P}_{\mathfrak{f}}=\{\mathfrak{p} \in \mathfrak{P} \mid \mathfrak{f} \subset \partial \mathfrak{p}\}$. Observing that the boundary of each diamond $\mathfrak{p}_{e, c}$ is composed of 4 sub-faces in $\mathfrak{F}_{c}$, exchanging the sums yields

$$
\mathrm{s}_{\boldsymbol{\beta} ; c}\left(\left(\zeta-\zeta_{c}\right) \mathrm{v},\left(\zeta-\zeta_{c}\right) \mathrm{v}\right) \leq 8 C_{T}^{2}\|\boldsymbol{\beta}\|_{L^{\infty}(c)} \sum_{\mathfrak{p} \in \mathfrak{P}_{c}} h_{c}^{-1}\left\|\mathbf{L}_{\mathcal{E}_{c}}\left(\left(\zeta-\zeta_{c}\right) \mathrm{v}\right)\right\|_{L^{2}(\mathfrak{p})}^{2}=8 C_{T}^{2}\|\boldsymbol{\beta}\|_{L^{\infty}(c)} h_{c}^{-1}\left\|\mathbf{L}_{\mathcal{E}_{c}}\left(\left(\zeta-\zeta_{c}\right) \mathrm{v}\right)\right\|_{L^{2}(c)}^{2} .
$$

Owing to upper bound from Proposition 4.1, the Lipschitz regularity of $\zeta$, and the definition of $\omega_{\zeta}$, we infer that

$$
\left.\mathrm{s}_{\boldsymbol{\beta} ; c}\left(\left(\zeta-\zeta_{c}\right) \mathrm{v},\left(\zeta-\zeta_{c}\right) \mathrm{v}\right) \leq 8 C_{T}^{2} C_{\sharp}^{2}\|\boldsymbol{\beta}\|_{\boldsymbol{L}^{\infty}(c)} h_{c}^{-1} \|\left(\zeta-\zeta_{c}\right) \mathrm{v}\right)\left\|_{2, c}^{2} \leq 8 C_{T}^{2} C_{\sharp}^{2} \omega_{\zeta}^{2} \tau^{-1}\right\| \mathrm{v} \|_{2, c}^{2} .
$$

Finally, collecting these two bounds leads to

$$
T_{2} \leq 2\left(\|\zeta\|_{L^{\infty}(\Omega)}^{2}+8 C_{T}^{2} C_{\sharp}^{2} \omega_{\zeta}^{2}\right)\|\mathrm{v}\|^{2} .
$$


(iii) Bound on $T_{3}$. We proceed as in the previous step (ii) to infer that

$$
T_{3} \leq 2\left(\|\zeta\|_{L^{\infty}(\Omega)}^{2}+n_{\mathrm{F}, \partial} C_{T}^{2} C_{\sharp}^{2} \omega_{\zeta}^{2}\right)\|\mathrm{v}\|^{2},
$$

where $n_{\mathrm{F}, \partial}=\left(\max _{c \in \mathrm{C}} \#\left(\mathrm{~F}_{c} \cap \mathrm{F}^{\partial}\right)\right)$ is the naximal number of boundary faces that a mesh cell can have.

(iv) Bound on $T_{4}$. To bound this last term, we use a different decomposition, namely

$$
T_{4}=\sum_{f \in \mathrm{F}^{\circ}} \mathrm{s}_{\zeta^{2} \boldsymbol{\beta} ; f}(\mathrm{v}, \mathrm{v})+\sum_{f \in \mathrm{F}^{\circ}} \Delta_{f}(\mathrm{v})=T_{4,1}+T_{4,2}
$$

with $\Delta_{f}(\mathrm{v})=\mathrm{s}_{\boldsymbol{\beta} ; f}(\zeta \mathrm{v}, \zeta \mathrm{v})-\mathbf{s}_{\zeta^{2} \boldsymbol{\beta} ; f}(\mathrm{v}, \mathrm{v})$. Observing that $\mathbf{s}_{\zeta^{2} \boldsymbol{\beta} ; f}(\mathrm{v}, \mathrm{v}) \leq\|\zeta\|_{L^{\infty}(f)}^{2} \mathbf{s}_{\boldsymbol{\beta} ; f}(\mathrm{v}, \mathrm{v})$ for all $f \in \mathrm{F}^{\circ}$, it follows that $T_{4,1} \leq\|\zeta\|_{L^{\infty}(\Omega)}^{2}\|v\|^{2}$. To bound the second term $T_{4,2}$, we recall the quantity $\boldsymbol{\delta}(\mathrm{v})$ defined by (4.7) and we obtain

$$
\Delta_{f}(\mathrm{v})=\int_{f}\left|\boldsymbol{\beta} \cdot \boldsymbol{n}_{f}\right|\left(\left(\llbracket \boldsymbol{\delta}(\mathrm{v}) \rrbracket+\zeta \llbracket \mathbf{L}_{\mathcal{E}}(\mathrm{v}) \rrbracket\right)^{2}-\zeta^{2} \llbracket \mathbf{L}_{\mathcal{E}}(\mathrm{v}) \rrbracket^{2}\right) .
$$

Then, applying Young's inequality and the trace inequality (4.8b) yields

$$
\begin{aligned}
\left|\Delta_{f}(\mathrm{v})\right| & \leq 2 \int_{f}\left|\boldsymbol{\beta} \cdot \boldsymbol{n}_{f}\right| \llbracket \boldsymbol{\delta}(\mathrm{v}) \rrbracket^{2}+\int_{f}\left|\boldsymbol{\beta} \cdot \boldsymbol{n}_{f}\right| \zeta^{2} \llbracket \mathbf{L}_{\mathcal{E}}(\mathrm{v}) \rrbracket^{2} \\
& \leq 4\left(2 C_{T} C_{\sharp} L_{\zeta}\right)^{2}\|\boldsymbol{\beta}\|_{\boldsymbol{L}^{\infty}(f)} \sum_{c \in \mathrm{C}_{f}} h_{c}\|\mathrm{v}\|_{2, c}^{2}+\|\zeta\|_{L^{\infty}(f)}^{2} \mathrm{~s}_{\boldsymbol{\beta} ; f}(\mathrm{v}, \mathrm{v}) .
\end{aligned}
$$

As a result, since $\# \mathrm{C}_{f}=2$ for all $f \in \mathrm{F}^{\circ}$ and introducing $\omega_{\zeta}$, we infer that

$$
T_{4,2} \leq 32 C_{T}^{2} C_{\sharp}^{2} \omega_{\zeta}^{2} \tau^{-1}\|\mathrm{v}\|_{2}^{2}+\|\zeta\|_{L^{\infty}(\Omega)}^{2} \sum_{f \in \mathrm{F}^{\circ}} \mathrm{s}_{\boldsymbol{\beta} ; f}(\mathrm{v}, \mathrm{v}) \leq\left(32 C_{T}^{2} C_{\sharp}^{2} \omega_{\zeta}^{2}+\|\zeta\|_{L^{\infty}(\Omega)}^{2}\right)\|\mathrm{v}\|^{2},
$$

whence

$$
T_{4} \leq 2\left(16 C_{T}^{2} C_{\sharp}^{2} \omega_{\zeta}^{2} L_{\zeta} h+\|\zeta\|_{L^{\infty}(\Omega)}^{2}\right)\|\mathrm{v}\|^{2} .
$$

(v) Conclusion. The expected inequality then follows from the above four bounds.

Proof of Proposition 4.7. Let $\vee \in \mathcal{E}$ and define

$$
\mathbb{S}=\sup _{\mathrm{w} \in \mathcal{E} \backslash\{0\}} \frac{\mathbb{A}_{\boldsymbol{\beta}, \boldsymbol{\mu}}(\mathrm{v}, \mathrm{w})}{\|\mathrm{w}\|} .
$$

Let us take $w=\zeta v+\theta v$ with $\theta>0$ to be chosen below. We infer from Lemma 6.1 that

$$
\mathbb{A}_{\boldsymbol{\beta}, \boldsymbol{\mu}}(\mathrm{v}, \mathrm{w}) \leq \mathbb{S}\|\mathrm{w}\| \leq \mathbb{S}\left(\theta+C_{\zeta}\left(\|\zeta\|_{L^{\infty}(\Omega)}+\omega_{\zeta}\right)\right)\|\mathrm{v}\|,
$$

so that it remains to prove that $\mathbb{A}_{\boldsymbol{\beta}, \boldsymbol{\mu}}(\mathrm{v}, \mathrm{w}) \gtrsim\|\mathrm{v}\|^{2}$. First, we split $\mathbb{A}_{\boldsymbol{\beta}, \boldsymbol{\mu}}$ as follows:

$$
\mathbb{A}_{\boldsymbol{\beta}, \boldsymbol{\mu}}(\mathrm{v}, \mathrm{w})=\mathbb{A}_{\boldsymbol{\beta},-\nabla \boldsymbol{\beta}^{\mathrm{T}}+\frac{1}{2}(\nabla \cdot \boldsymbol{\beta}) \mathbf{I d}}(\mathrm{v}, \mathrm{w})+\mathrm{H}_{\boldsymbol{\mu}+\nabla \boldsymbol{\beta}^{\mathrm{T}}-\frac{1}{2}(\nabla \cdot \boldsymbol{\beta}) \mathbf{I d}}(\mathrm{v}, \mathrm{w})=T_{1}+T_{2},
$$

where the bilinear form $\mathrm{H}_{\boldsymbol{\alpha}}$ is defined on $\mathcal{E} \times \mathcal{E}$ by

$$
\mathrm{H}_{\boldsymbol{\alpha}}(\mathrm{v}, \mathrm{w})=\sum_{c \in \mathrm{C}} \int_{c} \boldsymbol{\alpha} \mathbf{L}_{\mathcal{E}_{c}}(\mathrm{v}) \cdot \mathbf{L}_{\mathcal{E}_{c}}(\mathrm{w}),
$$

for all $\boldsymbol{\alpha} \in \boldsymbol{L}^{\infty}(\Omega)$. Let us bound from below the two terms $T_{1}$ and $T_{2}$. 
(i) Bound on $T_{1}$. We bound from below this term by considering the following decomposition

$$
\begin{aligned}
T_{1}=\mathbb{A}_{\boldsymbol{\beta},-\nabla \boldsymbol{\beta}^{\mathrm{T}}+\frac{1}{2}(\nabla \cdot \boldsymbol{\beta}) \mathbf{I d}}(\mathrm{v}, \mathrm{w})= & \mathbb{A}_{\zeta \boldsymbol{\beta},-\nabla(\zeta \boldsymbol{\beta})^{\mathrm{T}}+\frac{1}{2} \zeta(\nabla \cdot \boldsymbol{\beta}) \mathbf{I} \mathbf{d}}(\mathrm{v}, \mathrm{v}) \\
& +\mathbb{A}_{\boldsymbol{\beta},-\nabla \boldsymbol{\beta}^{\mathrm{T}}}(\mathrm{v}, \zeta \mathrm{v})-\mathbb{A}_{\zeta \boldsymbol{\beta},-\nabla(\zeta \boldsymbol{\beta})^{\mathrm{T}}}(\mathrm{v}, \mathrm{v}) \\
& +\mathrm{H}_{\frac{1}{2}(\nabla \cdot \boldsymbol{\beta}) \mathbf{I d}}(\mathrm{v}, \zeta \mathrm{v})-\mathrm{H}_{\frac{1}{2} \zeta(\nabla \cdot \boldsymbol{\beta}) \mathbf{I d}}(\mathrm{v}, \mathrm{v}) \\
& +\theta \mathbb{A}_{\boldsymbol{\beta},-\nabla \boldsymbol{\beta}^{\mathrm{T}}+\frac{1}{2}(\nabla \cdot \boldsymbol{\beta}) \mathbf{I d}}(\mathrm{v}, \mathrm{v})=T_{1,1}+T_{1,2}+T_{1,3}+T_{1,4}
\end{aligned}
$$

Regarding $T_{1,1}$, we use the relation (6.2) to infer that

$$
\begin{aligned}
T_{1,1} & =\frac{1}{2}\left(\sum_{c \in \mathrm{C}} \int_{c} \mathbf{L}_{\mathcal{E}_{c}}(\mathrm{v}) \cdot \boldsymbol{\sigma}_{\zeta \boldsymbol{\beta},-\nabla(\zeta \boldsymbol{\beta})^{\mathrm{T}}+\frac{1}{2} \zeta(\nabla \cdot \boldsymbol{\beta}) \mathbf{I d}} \cdot \mathbf{L}_{\mathcal{E}_{c}}(\mathrm{v})+\mathrm{A}_{|\zeta \boldsymbol{\beta} \cdot \boldsymbol{n}|}^{\partial}(\mathrm{v}, \mathrm{v})+\mathrm{s}_{\zeta \boldsymbol{\beta}}(\mathrm{v}, \mathrm{v})\right) \\
& \geq \frac{1}{2}\left(\sum_{c \in \mathrm{C}} \int_{c} \mathbf{L}_{\mathcal{E}_{c}}(\mathrm{v}) \cdot \boldsymbol{\sigma}_{\zeta \boldsymbol{\beta},-\nabla(\zeta \boldsymbol{\beta})^{\mathrm{T}}+\frac{1}{2} \zeta(\nabla \cdot \boldsymbol{\beta}) \mathbf{I d}} \cdot \mathbf{L}_{\mathcal{E}_{c}}(\mathrm{v})+\mathrm{A}_{|\boldsymbol{\beta} \cdot \boldsymbol{n}|}^{\partial}(\mathrm{v}, \mathrm{v})+\mathrm{s}_{\boldsymbol{\beta}}(\mathrm{v}, \mathrm{v})\right)
\end{aligned}
$$

since $\zeta \geq 1$. Then, observing that $\boldsymbol{\sigma}_{\zeta \boldsymbol{\beta},-\nabla(\zeta \boldsymbol{\beta})^{\mathrm{T}}+\frac{1}{2} \zeta(\nabla \cdot \boldsymbol{\beta}) \mathbf{I d}}=-\boldsymbol{\beta} \cdot \nabla \zeta \mathbf{I d}$ and using hypothesis $(\mathbf{H 2})$ together with the lower bound from Proposition (4.1), we infer that $T_{1,1} \geq \frac{1}{2}\|\mathrm{v}\|^{2}$. The next step consists in bounding the perturbation term $T_{1,2}$. To do so, we recall the identity (3.7) for $\mathrm{g}_{\boldsymbol{\beta}, \boldsymbol{\mu} ; c}$, and we observe that $\mathrm{g}_{\boldsymbol{\beta},-\nabla \boldsymbol{\beta}^{\mathrm{T}} ; c} \equiv 0$ and $\mathrm{g}_{\zeta \boldsymbol{\beta},-\nabla(\zeta \boldsymbol{\beta})^{\mathrm{T}} ; c} \equiv 0$, so that $T_{1,2}$ solely consists of surfacic terms:

$$
T_{1,2}=\left(\mathrm{n}_{\boldsymbol{\beta}}(\mathrm{v}, \zeta \mathrm{v})-\mathrm{n}_{\zeta \boldsymbol{\beta}}(\mathrm{v}, \mathrm{v})\right)+\left(\mathrm{s}_{\boldsymbol{\beta}}(\mathrm{v}, \zeta \mathrm{v})-\mathrm{s}_{\zeta \boldsymbol{\beta}}(\mathrm{v}, \mathrm{v})\right)+\left(\mathrm{A}_{(\boldsymbol{\beta} \cdot \boldsymbol{n})^{-}}^{\partial}(\mathrm{v}, \zeta \mathrm{v})-\mathrm{A}_{(\zeta \boldsymbol{\beta} \cdot \boldsymbol{n})^{-}}^{\partial}(\mathrm{v}, \mathrm{v})\right)
$$

Now, introducing the function $\boldsymbol{\delta}(\mathrm{v})$ locally defined by (4.7) and recalling that $\boldsymbol{\beta} \in \boldsymbol{W}^{1, \infty}(\Omega), \zeta \geq 1$, and $\zeta \in W^{1, \infty}(\Omega)$, so that $\zeta\left\{\left\{\mathbf{L}_{\mathcal{E}_{c}}(\mathrm{v})\right\}\right\}=\left\{\left[\zeta \mathbf{L}_{\mathcal{E}_{c}}(\mathrm{v})\right\}\right.$, we observe that

$$
\begin{aligned}
& \mathrm{n}_{\boldsymbol{\beta} ; x}(\mathrm{v}, \zeta \mathrm{v})-\mathrm{n}_{\zeta \boldsymbol{\beta} ; x}(\mathrm{v}, \mathrm{v})=\sum_{\mathfrak{f} \in \mathfrak{F}_{x}} \int_{\mathfrak{f}}\left(\boldsymbol{\beta} \cdot \boldsymbol{n}_{\mathfrak{f}}\right) \llbracket \mathbf{L}_{\mathcal{E}}(\mathrm{v}) \rrbracket \cdot\{\boldsymbol{\delta}(\mathrm{v})\}, \\
& \mathrm{s}_{\boldsymbol{\beta} ; x}(\mathrm{v}, \zeta \mathrm{v})-\mathrm{s}_{\zeta \boldsymbol{\beta} ; x}(\mathrm{v}, \mathrm{v})=\sum_{\mathfrak{f} \in \mathfrak{F}_{x}} \int_{\mathfrak{f}}\left|\boldsymbol{\beta} \cdot \boldsymbol{n}_{\mathfrak{f}}\right| \llbracket \mathbf{L}_{\mathcal{E}}(\mathrm{v}) \rrbracket \cdot \llbracket \boldsymbol{\delta}(\mathrm{v}) \rrbracket
\end{aligned}
$$

for all $x \in \mathrm{F}^{\circ}$ or $x \in \mathrm{C}$, and

$$
\mathrm{A}_{(\boldsymbol{\beta} \cdot \boldsymbol{n})^{-}, f}^{\partial}(\mathrm{v}, \zeta \mathrm{v})-\mathrm{A}_{(\zeta \boldsymbol{\beta} \cdot \boldsymbol{n})^{-}, f}^{\partial}(\mathrm{v}, \mathrm{v})=\int_{f}(\boldsymbol{\beta} \cdot \boldsymbol{n})^{-} \mathbf{L}_{\mathcal{E}}(\mathrm{v}) \cdot \boldsymbol{\delta}(\mathrm{v})
$$

for all $f \in \mathrm{F}^{2}$. Then, applying the Cauchy-Schwarz inequality to these three terms yields

$$
T_{1,2} \leq 6\left(|\mathrm{v}|_{\partial}^{2}+|\mathrm{v}|_{\mathrm{s}}^{2}\right)^{\frac{1}{2}}\left(2 \sum_{c \in \mathrm{C}}\|\boldsymbol{\beta}\|_{\boldsymbol{L}^{\infty}(c)} \sum_{\mathfrak{p} \in \mathfrak{P}_{c}}\|\boldsymbol{\delta}(\mathrm{v})\|_{L^{2}(\partial \mathfrak{p})}^{2}\right)^{\frac{1}{2}}
$$

In addition, observing that $\boldsymbol{\sigma}_{\boldsymbol{\beta},-\nabla \boldsymbol{\beta}^{\mathrm{T}}+\frac{1}{2}(\nabla \cdot \boldsymbol{\beta}) \mathbf{I d}} \equiv \mathbf{0}$ and using the identity (6.2), we have

$$
\mathbb{A}_{\boldsymbol{\beta},-\nabla \boldsymbol{\beta}^{\mathrm{T}}+\frac{1}{2}(\nabla \cdot \boldsymbol{\beta}) \mathbf{I d}}(\mathrm{v}, \mathrm{v})=\frac{1}{2}\left(|\mathrm{v}|_{\partial}^{2}+|\mathrm{v}|_{\mathrm{s}}^{2}\right)
$$

so that combining this expression with the above estimate yields

$$
T_{1,2} \leq 12\left(\mathbb{A}_{\boldsymbol{\beta},-\nabla \boldsymbol{\beta}^{\mathrm{T}}+\frac{1}{2}(\nabla \cdot \boldsymbol{\beta}) \mathbf{I d}}(\mathrm{v}, \mathrm{v})\right)^{\frac{1}{2}}\left(\sum_{c \in \mathrm{C}}\|\boldsymbol{\beta}\|_{\boldsymbol{L}^{\infty}(c)} \sum_{\mathfrak{p} \in \mathfrak{P}_{c}}\|\boldsymbol{\delta}(\mathrm{v})\|_{\boldsymbol{L}^{2}(\partial \mathfrak{p})}^{2}\right)^{\frac{1}{2}}
$$


Finally, we use the inequalities (4.8a)-(4.8b) together with the definition of $\omega_{\zeta}$, to infer that

$$
T_{1,2} \leq C_{\boldsymbol{\delta}} \omega_{\zeta}\left(\mathbb{A}_{\boldsymbol{\beta},-\nabla \boldsymbol{\beta}^{\mathrm{T}}+\frac{1}{2}(\nabla \cdot \boldsymbol{\beta}) \mathbf{I d}}(\mathrm{v}, \mathrm{v})\right)^{\frac{1}{2}}\left(\tau^{-1}\|\mathbf{v}\|_{2}^{2}\right)^{\frac{1}{2}},
$$

where $C_{\boldsymbol{\delta}}>0$ depends exclusively on the numerical constants $C_{\mathrm{T}}$ and $C_{\sharp}$. Now, we collect the bounds on $T_{1,1}$ and $T_{1,2}$ and we apply Young's inequality to obtain

$$
\mathbb{A}_{\boldsymbol{\beta},-\nabla \boldsymbol{\beta}^{\mathrm{T}}+\frac{1}{2}(\nabla \cdot \boldsymbol{\beta}) \mathbf{I d}}(\mathrm{v}, \mathrm{w}) \geq \frac{1}{4}\|\mathbf{v}\|^{2}+\left(\theta-C_{\boldsymbol{\delta}}^{2} \omega_{\zeta}^{2}\right) \mathbb{A}_{\boldsymbol{\beta},-\nabla \boldsymbol{\beta}^{\mathrm{T}}+\frac{1}{2}(\nabla \cdot \boldsymbol{\beta}) \mathbf{I d}}(\mathrm{v}, \mathrm{v})+T_{1,3}
$$

As a result, choosing $\theta=C_{\delta}^{2} \omega_{\zeta}^{2}$ yields

$$
\mathbb{A}_{\boldsymbol{\beta},-\nabla \boldsymbol{\beta}^{\mathrm{T}}+\frac{1}{2}(\nabla \cdot \boldsymbol{\beta}) \mathbf{I d}}(\mathrm{v}, \mathrm{w}) \geq \frac{1}{4}\|\mathrm{v}\|^{2}+T_{1,3} .
$$

(ii) Bound on $T_{2}$. First, we rewrite this term as:

$$
\begin{aligned}
T_{2}= & \theta \mathrm{H}_{\boldsymbol{\mu}+\nabla \boldsymbol{\beta}^{\mathrm{T}}-\frac{1}{2}(\nabla \cdot \boldsymbol{\beta}) \mathbf{I d}}(\mathrm{v}, \mathrm{v}) \\
& +\mathrm{H}_{\zeta\left(\boldsymbol{\mu}+\nabla \boldsymbol{\beta}^{\mathrm{T}}-\frac{1}{2}(\nabla \cdot \boldsymbol{\beta}) \mathbf{I d}\right)}(\mathrm{v}, \mathrm{v}) \\
& +\mathrm{H}_{\boldsymbol{\mu}+\nabla \boldsymbol{\beta}^{\mathrm{T}}-\frac{1}{2}(\nabla \cdot \boldsymbol{\beta}) \mathbf{I d}}(\mathrm{v}, \zeta \mathrm{v})-\mathrm{H}_{\zeta\left(\boldsymbol{\mu}+\nabla \boldsymbol{\beta}^{\mathrm{T}}-\frac{1}{2}(\nabla \cdot \boldsymbol{\beta}) \mathbf{I d}\right)}(\mathrm{v}, \mathrm{v})=T_{2,1}+T_{2,2}+T_{2,3} .
\end{aligned}
$$

Concerning $T_{2,1}$, we have

$$
T_{2,1}=\frac{\theta}{2} \sum_{c \in \mathrm{C}} \int_{c} \boldsymbol{\sigma}_{\boldsymbol{\beta}, \boldsymbol{\mu}} \mathbf{L}_{\mathcal{E}_{c}}(\mathrm{v}) \cdot \mathbf{L}_{\mathcal{E}_{c}}(\mathrm{v}) \geq \frac{\theta \lambda_{b}}{2} \sum_{c \in \mathrm{C}}\left\|\mathbf{L}_{\mathcal{E}_{c}}(\mathrm{v})\right\|_{\boldsymbol{L}^{2}(c)}^{2} \geq \frac{C_{\sharp}^{2} \theta \lambda_{b}}{2}\|\mathrm{v}\|_{2}^{2},
$$

where we have used hypothesis $(\mathbf{H 2})$ (recall that $\lambda_{b} \leq 0$ ) and the upper bound from Proposition 4.1. The second term $T_{2,2}$ is treated similarly:

$$
T_{2,2}=\frac{1}{2} \sum_{c \in \mathrm{C}} \int_{c} \zeta \boldsymbol{\sigma}_{\boldsymbol{\beta}, \boldsymbol{\mu}} \mathbf{L}_{\mathcal{E}_{c}}(\mathrm{v}) \cdot \mathbf{L}_{\mathcal{E}_{c}}(\mathrm{v}) \geq \frac{C_{\sharp}^{2} \lambda_{b}}{2}\|\zeta\|_{L^{\infty}(\Omega)}\|\mathrm{v}\|_{2}^{2} .
$$

Collecting these bounds yields

$$
T_{2} \geq \frac{\lambda_{b}}{2} \vartheta\|\mathrm{u}\|_{2}^{2}+T_{2,3}
$$

with $\vartheta=C_{\sharp}^{2}\left(\theta+\|\zeta\|_{L^{\infty}(\Omega)}\right)$.

(iii) Bound on $T_{1}+T_{2}$. Collecting the estimates (6.3) and (6.4), we obtain

$$
\mathbb{A}_{\boldsymbol{\beta}, \boldsymbol{\mu}}(\mathrm{v}, \mathrm{w}) \geq \frac{1}{4}\|\mathrm{v}\|^{2}+\frac{\tau \lambda_{b}}{2} \vartheta \tau^{-1}\|\mathrm{v}\|_{2}^{2}+T_{1,3}+T_{2,3} .
$$

We observe that

$$
\begin{aligned}
T_{1,3}+T_{2,3} & =\mathrm{H}_{\frac{1}{2}(\nabla \cdot \boldsymbol{\beta}) \mathbf{I d}}(\mathrm{v}, \zeta \mathrm{v})-\mathrm{H}_{\frac{1}{2} \zeta(\nabla \cdot \boldsymbol{\beta}) \mathbf{I d}}(\mathrm{v}, \mathrm{v})+\mathrm{H}_{\boldsymbol{\mu}+\nabla \boldsymbol{\beta}^{\mathrm{T}}-\frac{1}{2}(\nabla \cdot \boldsymbol{\beta}) \mathbf{I d}}(\mathrm{v}, \zeta \mathrm{v})-\mathrm{H}_{\zeta\left(\boldsymbol{\mu}+\nabla \boldsymbol{\beta}^{\mathrm{T}}-\frac{1}{2}(\nabla \cdot \boldsymbol{\beta}) \mathbf{I d}\right)}(\mathrm{v}, \mathrm{v}) \\
& =\mathrm{H}_{\boldsymbol{\mu}+\nabla \boldsymbol{\beta}^{\mathrm{T}}}(\mathrm{v}, \zeta \mathrm{v})-\mathrm{H}_{\zeta\left(\boldsymbol{\mu}+\nabla \boldsymbol{\beta}^{\mathrm{T}}\right)}(\mathrm{v}, \mathrm{v})=\sum_{c \in \mathrm{C}} \int_{c}\left(\boldsymbol{\mu}+\nabla \boldsymbol{\beta}^{\mathrm{T}}\right) \mathbf{L}_{\mathcal{E}_{c}}(\mathrm{v}) \cdot \boldsymbol{\delta}(\mathrm{v}) .
\end{aligned}
$$

Applying successively the Hölder inequality, the inequality (4.8a) and the upper bound from Proposition 4.1, we infer that

$$
\left|T_{1,3}+T_{2,3}\right| \leq \sum_{c \in \mathrm{C}}\left\|\boldsymbol{\mu}+\nabla \boldsymbol{\beta}^{\mathrm{T}}\right\|_{\boldsymbol{L}^{\infty}(c)}\left\|\mathbf{L}_{\mathcal{E}}(\mathrm{v})\right\|_{\boldsymbol{L}^{2}(c)}\|\boldsymbol{\delta}(\mathrm{v})\|_{\boldsymbol{L}^{2}(c)} \leq\left\|\boldsymbol{\mu}+\nabla \boldsymbol{\beta}^{\mathrm{T}}\right\|_{\boldsymbol{L}^{\infty}(\Omega)} C_{\sharp}^{2} L_{\zeta} h\|\mathrm{v}\|_{2}^{2} .
$$


As a result, we obtain

$$
\mathbb{A}_{\boldsymbol{\beta}, \boldsymbol{\mu}}(\mathrm{v}, \mathrm{w}) \geq \frac{1}{4}\|\mathrm{v}\|^{2}+\left(\frac{\tau \lambda_{b}}{2} \vartheta-\frac{h}{4 h_{0}}\right) \tau^{-1}\|\mathrm{v}\|_{2}^{2},
$$

with the reference length $h_{0}=\left(4 C_{\sharp}^{2}\left\|\boldsymbol{\mu}+\nabla \boldsymbol{\beta}^{\mathrm{T}}\right\|_{\boldsymbol{L}^{\infty}(\Omega)} \tau L_{\zeta}\right)^{-1}$. Hence, there exists $\varrho^{\prime}>0$ such that $\mathbb{A}_{\boldsymbol{\beta}, \boldsymbol{\mu}}(\mathrm{v}, \mathrm{w}) \geq$ $\varrho^{\prime}\|\mathrm{v}\|^{2}$, as soon as $\lambda_{b}$ and $h$ satisfy (4.6).

\subsection{Bound on consistency error and a priori estimate}

Proof of Lemma 4.9. Let $\boldsymbol{y}_{\mid c}=\left(\boldsymbol{u}-\mathcal{I}_{\mathcal{E}}(\boldsymbol{u})\right)_{\mid c}$ for all $c \in$ C. Note that $\boldsymbol{y}_{\mid \partial \mathfrak{p}} \in \boldsymbol{L}^{q}(\partial \mathfrak{p})$ for all $\mathfrak{p} \in \mathfrak{P}_{c}$. Let $\boldsymbol{v} \in \mathcal{E}$. Owing to the definitions of $\mathbb{A}_{\boldsymbol{\beta}, \boldsymbol{\mu}}$ and $\Sigma$, it follows that $\Sigma\left(\boldsymbol{s}, \boldsymbol{u}_{D} ; \mathrm{v}\right)-\mathbb{A}_{\boldsymbol{\beta}, \boldsymbol{\mu}}\left(\mathrm{R}_{\mathcal{E}}(\boldsymbol{u}), \mathrm{v}\right)=T_{1}+T_{2}+T_{3}+T_{4}$, with

$$
\begin{aligned}
& T_{1}:=\sum_{c \in \mathrm{C}} \int_{c}\left(\nabla \boldsymbol{\beta}+\boldsymbol{\mu}^{\mathrm{T}}-(\nabla \cdot \boldsymbol{\beta}) \mathbf{I d}\right) \mathbf{L}_{\mathcal{E}_{c}}(\mathrm{v}) \cdot \boldsymbol{y}, \quad T_{2}:=\sum_{\mathrm{X} \in\left\{\mathrm{F}^{\circ}, \mathrm{C}\right\}} \sum_{x \in \mathrm{X}} \sum_{\mathfrak{f} \in \mathfrak{F}_{x}} \int_{\mathfrak{f}}(\boldsymbol{\beta} \cdot \boldsymbol{n}) \llbracket \mathbf{L}_{\mathcal{E}}(\mathrm{v}) \rrbracket \cdot\{\boldsymbol{y}\}, \\
& T_{3}:=\sum_{\mathrm{X} \in\left\{\mathrm{F}^{\circ}, \mathrm{C}\right\}} \sum_{x \in \mathrm{X}} \sum_{\mathfrak{f} \in \mathfrak{F}_{x}} \int_{\mathfrak{f}}|\boldsymbol{\beta} \cdot \boldsymbol{n}| \llbracket \mathbf{L}_{\mathcal{E}}(\mathrm{v}) \rrbracket \cdot \llbracket \boldsymbol{y} \rrbracket \text { and } \quad T_{4}:=\sum_{f \in \mathrm{F}^{\partial}} \sum_{\mathfrak{f} \in \mathfrak{F}_{f}^{\partial}} \int_{\mathfrak{f}}(\boldsymbol{\beta} \cdot \boldsymbol{n})^{-} \mathbf{L}_{\mathcal{E}_{\mathcal{C}_{f}}}(\mathrm{v}) \cdot \boldsymbol{y} .
\end{aligned}
$$

Indeed, the first term $T_{1}$ is obtained using the definition (3.7) of $\mathbf{g}_{\boldsymbol{\beta}, \boldsymbol{\mu} ; \boldsymbol{c}}$ together with the following integration by part formula (6.1) and

$$
\sum_{\mathfrak{p} \in \mathfrak{P}_{c}} \int_{\mathfrak{p}}((\boldsymbol{\beta} \cdot \nabla) \boldsymbol{y}) \cdot \mathbf{L}_{\mathcal{E}_{c}}(\mathrm{v})=-\sum_{\mathfrak{p} \in \mathfrak{P}_{c}} \int_{\mathfrak{p}}\left((\boldsymbol{\beta} \cdot \nabla) \mathbf{L}_{\mathcal{E}_{c}}(\mathrm{v})\right) \cdot \boldsymbol{y}-\int_{c}(\nabla \cdot \boldsymbol{\beta}) \mathbf{L}_{\mathcal{E}_{c}}(\mathrm{v}) \cdot \boldsymbol{y}+\sum_{\mathfrak{p} \in \mathfrak{P}_{c}} \int_{\mathfrak{p}} \nabla \cdot\left(\boldsymbol{\beta} \boldsymbol{y} \cdot \mathbf{L}_{\mathcal{E}_{c}}(\mathrm{v})\right),
$$

holding for all $c \in \mathrm{C}$ and all $\vee \in \mathcal{E}_{c}$. The terms $T_{2}$ and $T_{3}$ result from the rightmost term of the relation (6.1) and the fact that $(\boldsymbol{\beta} \cdot \boldsymbol{n}) \llbracket \boldsymbol{u} \rrbracket_{\mid \mathfrak{f}} \equiv 0$ for all $\mathfrak{f} \in \mathfrak{F}_{x}$. Finally, the term $T_{4}$ is inferred observing that $\boldsymbol{u}_{D}=\boldsymbol{u}_{\mid \partial \Omega}$. It remains to bound these four terms. First, let us consider $T_{1}$. Let $q \in[1,2]$ and denote $q^{\prime} \geq 2$ its conjugate number, i.e., $1=1 / q+1 / q^{\prime}$. From the Hölder inequality, we infer that

$$
\left|\int_{c}\left(\nabla \boldsymbol{\beta}+\boldsymbol{\mu}^{\mathrm{T}}-(\nabla \cdot \boldsymbol{\beta}) \mathbf{I} \mathbf{d}\right) \mathbf{L}_{\mathcal{E}_{c}}(\mathrm{v}) \cdot \boldsymbol{y}\right| \leq N_{\infty}\|\boldsymbol{y}\|_{\boldsymbol{L}^{q}(c)}\left\|\mathbf{L}_{\mathcal{E}_{c}}(\mathrm{v})\right\|_{\boldsymbol{L}^{q^{\prime}}(c)},
$$

with $N_{\infty}=\left\|\nabla \boldsymbol{\beta}+\boldsymbol{\mu}^{\mathrm{T}}-\nabla \cdot \boldsymbol{\beta} \mathbf{I d}\right\|_{\boldsymbol{L}^{\infty}(c)}$. Then, using a local inverse inequality (see [13], Lem. 1.138), we infer that

$$
\left|\int_{c}\left(\nabla \boldsymbol{\beta}+\boldsymbol{\mu}^{\mathrm{T}}-(\nabla \cdot \boldsymbol{\beta}) \mathbf{I d}\right) \mathbf{L}_{\mathcal{E}_{c}}(\mathrm{v}) \cdot \boldsymbol{y}\right| \leq N_{\infty} h_{c}^{\theta}\|\boldsymbol{y}\|_{\boldsymbol{L}^{q}(c)}\left\|\mathbf{L}_{\mathcal{E}_{c}}(\mathrm{v})\right\|_{\boldsymbol{L}^{2}(c)},
$$

with $\theta=d\left(\frac{1}{2}-\frac{1}{q}\right)$, so that the Hölder inequality yields

$$
\left|\sum_{c \in \mathrm{C}} \int_{c}\left(\nabla \boldsymbol{\beta}+\boldsymbol{\mu}^{\mathrm{T}}-(\nabla \cdot \boldsymbol{\beta}) \mathbf{I} \mathbf{d}\right) \mathbf{L}_{\mathcal{E}_{c}}(\mathrm{v}) \cdot \boldsymbol{y}\right| \leq\left(\sum_{c \in \mathrm{C}} N_{\infty}^{q} h_{c}^{\theta q}\|\boldsymbol{y}\|_{\boldsymbol{L}^{q}(c)}^{q}\right)^{\frac{1}{q}}\left(\sum_{c \in \mathrm{C}}\left\|\mathbf{L}_{\mathcal{E}_{c}}(\mathrm{v})\right\|_{\boldsymbol{L}^{2}(c)}^{q^{\prime}}\right)^{\frac{1}{q^{\prime}}} .
$$

Moreover, recalling that $q^{\prime} \geq 2$ so that $|\cdot|_{\ell^{\prime}} \leq|\cdot|_{\ell^{2}}$, and using the upper bound in Proposition 4.1 leads to

$$
\left|T_{1}\right|=\left|\sum_{c \in \mathrm{C}} \int_{c}\left(\nabla \boldsymbol{\beta}+\boldsymbol{\mu}^{\mathrm{T}}-(\nabla \cdot \boldsymbol{\beta}) \mathbf{I} \mathbf{d}\right) \mathbf{L}_{\mathcal{E}_{c}}(\mathrm{v}) \cdot \boldsymbol{y}\right| \lesssim\left(\sum_{c \in \mathrm{C}} N_{\infty}^{q} h_{c}^{\theta q}\|\boldsymbol{y}\|_{\boldsymbol{L}^{q}(c)}^{q}\right)^{\frac{1}{q}}\|\mathrm{v}\|_{2} .
$$

To bound the two terms $T_{2}$ and $T_{3}$, we consider a sub-face $\mathfrak{f} \in \mathfrak{F}_{x}$ for all $x \in \mathrm{X}$ with $\mathrm{X} \in\left\{\mathrm{F}^{\circ}, \mathrm{C}\right\}$. As above, the Hölder inequality yields

$$
\mid \int_{\mathfrak{f}}\left(\boldsymbol{\beta} \cdot \boldsymbol{n}_{\mathfrak{f}}\right) \llbracket \mathbf{L}_{\mathcal{E}}(\mathrm{v}) \rrbracket \cdot\left\{\{ \boldsymbol { y } \} \left|\leq\|\boldsymbol{\beta}\|_{\boldsymbol{L}^{\infty}(\mathfrak{f})}^{\frac{1}{2}}\|\{\{\boldsymbol{y}\}\}\|_{\boldsymbol{L}^{q}(\mathfrak{f})}\left\|\left|\boldsymbol{\beta} \cdot \boldsymbol{n}_{\mathfrak{f}}\right|^{\frac{1}{2}} \llbracket \mathbf{L}_{\mathcal{E}}(\mathrm{v}) \rrbracket\right\|_{\boldsymbol{L}^{q^{\prime}}(\mathfrak{f})},\right.\right.
$$


so that using a local inverse inequality, we obtain

$$
\mid \int_{\mathfrak{f}}\left(\boldsymbol{\beta} \cdot \boldsymbol{n}_{\mathfrak{f}}\right) \llbracket \mathbf{L}_{\mathcal{E}}(\mathrm{v}) \rrbracket \cdot\left\{\{\boldsymbol{y}\} \mid \leq h_{\mathfrak{f}}^{\theta^{\prime}}\|\boldsymbol{\beta}\|_{\boldsymbol{L}^{\infty}(\mathfrak{f})}^{\frac{1}{2}} \|\left\{\{\boldsymbol{y}\}\left\|_{\boldsymbol{L}^{q}(\mathfrak{f})}\right\|\left|\left(\boldsymbol{\beta} \cdot \boldsymbol{n}_{\mathfrak{f}}\right)\right|^{\frac{1}{2}} \llbracket \mathbf{L}_{\mathcal{E}}(\mathrm{v}) \rrbracket \|_{\boldsymbol{L}^{2}(\mathfrak{f})},\right.\right.
$$

with $\theta^{\prime}=(d-1)\left(\frac{1}{q}-\frac{1}{2}\right)$. Hence, denoting $\sum_{\mathfrak{f}}=\sum_{\mathrm{X} \in\left\{\mathrm{F}^{\circ}, \mathrm{C}\right\}} \sum_{x \in \mathrm{X}} \quad \sum_{\mathfrak{f} \in \mathfrak{F}_{x}}$, it follows from the triangle inequality, the Hölder inequality and $q^{\prime} \geq 2$ that

$$
\mid \sum_{\mathfrak{f}} \int_{\mathfrak{f}}\left(\boldsymbol{\beta} \cdot \boldsymbol{n}_{\mathfrak{f}}\right) \llbracket \mathbf{L}_{\mathcal{E}}(\mathrm{v}) \rrbracket \cdot\left\{\{\boldsymbol{y}\} \mid \leq\left(\sum_{\mathfrak{f}} h_{\mathfrak{f}}^{\theta^{\prime} q}\|\boldsymbol{\beta}\|_{\boldsymbol{L}^{\infty}(\mathfrak{f})}^{\frac{q}{2}} \|\left\{\{\boldsymbol{y}\} \|_{\boldsymbol{L}^{q}(\mathfrak{f})}^{q}\right)^{\frac{1}{q}}\left(\sum_{\mathfrak{f}}\left\|\left.\boldsymbol{\beta} \cdot \boldsymbol{n}_{\mathfrak{f}}\right|^{\frac{1}{2}} \llbracket \mathbf{L}_{\mathcal{E}}(\mathrm{v}) \rrbracket\right\|_{\boldsymbol{L}^{2}(\mathfrak{f})}^{2}\right)^{\frac{1}{2}} .\right.\right.
$$

Next, owing to the definitions (3.8) and (3.10) of $\mathrm{n}_{\boldsymbol{\beta}}$ and $\mathrm{s}_{\boldsymbol{\beta}}$ respectively, the mesh regularity and recalling the inequality $|a \pm b|^{q} \leq 2^{q-1}\left(|a|^{q}+|b|^{q}\right)$, we infer that

$$
\left|T_{2}+T_{3}\right| \lesssim\left(\sum_{c \in \mathrm{C}} \sum_{\mathfrak{p} \in \mathfrak{P}_{c}} h_{c}^{\theta^{\prime} q}\|\boldsymbol{\beta}\|_{\boldsymbol{L}^{\infty}(c)}^{\frac{q}{2}}\|\boldsymbol{y}\|_{\boldsymbol{L}^{q}(\partial \mathfrak{p})}^{q}\right)^{\frac{1}{q}} \mathrm{~s}_{\boldsymbol{\beta}}(\mathrm{v}, \mathrm{v})^{\frac{1}{2}}
$$

Finally, proceeding similarly, we also infer that

$$
\left|T_{4}\right| \lesssim\left(\sum_{f \in \mathrm{F}^{\partial}} h_{c_{f} \theta^{\prime} q}\|\boldsymbol{\beta}\|_{\boldsymbol{L}^{\infty}(f)}^{\frac{q}{2}}\|\boldsymbol{y}\|_{\boldsymbol{L}^{q}(f)}^{q}\right)^{\frac{1}{q}} \mathrm{~A}_{|\boldsymbol{\beta} \cdot \boldsymbol{n}|}^{\partial}(\mathrm{v}, \mathrm{v})^{\frac{1}{2}}
$$

and the expected result follows from the above bounds.

\section{REFERENCES}

[1] R. Abraham, J.E. Marsden and T. Ratiu, Manifolds, tensor analysis, and applications. Vol. 75 of Appl. Math. Sci. SpringerVerlag, New York, 2nd edition (1988).

[2] C. Amrouche, C. Bernardi, M. Dauge and V. Girault, Vector potentials in three-dimensional non-smooth domains. Math. Methods Appl. Sci. 21 (1998) 823-864.

[3] J. Bonelle, Compatible Discrete Operator schemes on polyhedral meshes for elliptic and Stokes equations. Ph.D. thesis, Université Paris Est (2014).

[4] J. Bonelle, D. Di Pietro and A. Ern, Low-order reconstruction operators on polyhedral meshes: application to compatible discrete operator schemes. Comput. Aided Geom. Design 35/36 (2015) 27-41.

[5] J. Bonelle and A. Ern, Analysis of compatible discrete operator schemes for elliptic problems on polyhedral meshes. ESAIM: M2AN 48 (2014) 553-581.

[6] J. Bonelle and A. Ern, Analysis of compatible discrete operator schemes for stokes problems on polyhedral meshes. IMA J. Numer. Anal. 34 (2014) 553-581.

[7] A. Bossavit, Extrusion, contraction: their discretization via Whitney forms. In Selected papers from: 10th International IGTE Symposium on Numerical Field Computation, Graz, 2002. COMPEL 22 (2003) 470-480.

[8] P. Cantin and A. Ern, Vertex-based compatible discrete operator schemes on polyhedral meshes for advection-diffusion equations. Comput. Meth. Appl. Math. 16 (2016) 187-212.

[9] S.H. Christiansen, A construction of spaces of compatible differential forms on cellular complexes. Math. Models Methods Appl. Sci. 18 (2008) 739-757.

[10] L. Codecasa, R. Specogna and F. Trevisan, A new set of basis functions for the discrete geometric approach. J. Comput. Phys. 229 (2010) 7401-7410.

[11] P. Deuring, R. Eymard and M. Mildner, $\mathrm{L}^{2}$-stability independent of diffusion for a Finite Element-Finite Volume discretization of a linear convection-diffusion equation. SIAM J. Numer. Anal. 53 (2015) 508-526.

[12] A. Devinatz, R. Ellis and A. Friedman. The asymptotic behavior of the first real eigenvalue of second order elliptic operators with a small parameter in the highest derivatives. II. Indiana Univ. Math. J. 23 (1973-1974) 991-1011.

[13] A. Ern and J.-L. Guermond, Theory and practice of finite elements. Vol. 159 of Applied Mathematical Sciences. Springer-Verlag, New York (2004). 
[14] A. Ern and J.-L. Guermond, Discontinuous Galerkin methods for Friedrichs' systems. I. General theory. SIAM J. Numer. Anal. 44 (2006) 753-778.

[15] A. Ern and J.-L. Guermond, Finite element quasi-interpolation and best approximation. ESAIM: M2AN 51 (2017) $1367-1385$.

[16] M. Gerritsma, An introduction to a compatible spectral discretization method. Mech. Adv. Mater. Struct. 19 (2012) 48-67.

[17] V. Girault, The Navier-Stokes Equations Theory and Numerical Methods. In: Proc. of a Conference held at Oberwolfach, 1988. Springer Berlin Heidelberg (1990) 201-218.

[18] H. Heumann, Eulerian and Semi-Lagrangian Methods for Advection-Diffusion of Differential Forms. Ph.D. thesis, ETH Zürich (2011).

[19] H. Heumann, R. Hiptmair and C. Pagliantini, Stabilized Galerkin for Transient Advection of Differential Forms. Research report, SAM, ETH Zürich (2015).

[20] C. Johnson and J. Pitkäranta, An analysis of the discontinuous Galerkin method for a scalar hyperbolic equation. Math. Comput. 46 (1986) 1-26.

[21] J. Kreeft, A. Palha and M. Gerritsma, Mimetic framework on curvilinear quadrilaterals of arbitrary order. Preprint arXiv: $1111.4304(2011)$

[22] P. Lesaint and P.-A. Raviart, On a finite element method for solving the neutron transport equation. In Mathematical Aspects of Finite Elements in Partial Differential Equations. Academic Press (1974) 89-123

[23] P. Mullen, A. McKenzie, D. Pavlov, L. Durant, Y. Tong, E. Kanso, J.E. Marsden and M. Desbrun, Discrete Lie advection of differential forms. Found. Comput. Math. 11 (2011) 131-149.

[24] A. Palha, High order mimetic discretization. PhD thesis, Technische Universiteit Delft (2013).

[25] S. Zaglmayr, High Order Finite Element Methods for Electromagnetic Field Computation. Ph.D. thesis, Johannes Kepler University (2006).

[26] G.M. Ziegler, Lectures on polytopes. Graduate texts in mathematics. Springer, New York (1995). 